\title{
LTE Network Enhancement for Vehicular Safety Communication
}

\author{
Wooseong Kim ${ }^{1}$ and Eun-Kyu Lee ${ }^{2}$ \\ ${ }^{1}$ Department of Computer Engineering, Gachon University, 1342 SeongNam-ro, Gyeonggi, Republic of Korea \\ ${ }^{2}$ Department of Information and Telecommunication Engineering, National University, 119 Academy-ro, \\ Yeonsu-gu, Incheon, Republic of Korea
}

Correspondence should be addressed to Wooseong Kim; wooseong@gachon.ac.kr and Eun-Kyu Lee; eklee@inu.ac.kr

Received 13 October 2016; Revised 8 January 2017; Accepted 15 January 2017; Published 19 February 2017

Academic Editor: Barbara M. Masini

Copyright (C) 2017 Wooseong Kim and Eun-Kyu Lee. This is an open access article distributed under the Creative Commons Attribution License, which permits unrestricted use, distribution, and reproduction in any medium, provided the original work is properly cited.

\begin{abstract}
Direct vehicle-to-vehicle (V2V) and vehicle-to-infrastructure (V2I) communications have been popularly considered for safe driving of manned or unmanned vehicles. The V2I communication is better than the V2V communication for propagating safety messages at critical regions like intersections where the safety messages must be delivered to surround vehicles with low latency and loss, since transmitters as infrastructure can have line of sight to the receiver vehicles and control wireless medium access in a centralized manner unlike V2V. Long-Term Evolution (LTE) cellular networks are rapidly deployed in the world with explosively increasing mobile traffic. As many automobile manufacturers choose LTE on-board devices for telematics, the LTE system can be utilized also for safety purposes instead of $802.11 \mathrm{p} /$ WAVE based roadside units (RSUs). Previous literatures have studied mostly current LTE system analysis in aspect of theoretical network capacity and end-to-end delay to investigate feasibility of V2I communication. In this paper, we propose new enhancement of a current LTE system specified by 3rd-Generation Partnership Project (3GPP) LTE standards while addressing major delay challenges. From simulation, we confirm that our three key solutions can reduce end-to-end delay effectively in the LTE system to satisfy requirements of safety message delivery.
\end{abstract}

\section{Introduction}

In the past, attempts have been made to introduce vehicular communication for Intelligent Transport Systems (ITS) using the $5.9 \mathrm{GHz}$ dedicate frequency spectrum, based on Dedicated Short-Range Communications (DSRC) and the IEEE 802.11p/Wireless Access for Vehicular Environments (WAVE) standards $[1,2]$. For example, the International Organization for Standardization (ISO) and European Telecommunications Standards Institute (ETSI) Cooperative Intelligent Transport Systems (C-ITS) standards elaborated several use cases regarding traffic control and road safety, not only for vehicle-to-vehicle $(\mathrm{V} 2 \mathrm{~V})$ direct communication between vehicles, but also for vehicle-to-infrastructure (V2I) indirect communication using roadside units (RSUs) along the road [3]. The United States Department of Transportation (USDOT) has issued an Advance Notice of Proposed Rule Making (NPRM) mandating the use of this technology for cars in the near future. Manufacturer-implemented standard hardware for the $\mathrm{V} 2 \mathrm{~V}$ direct communication inside cars is coming soon, but dedicated RSUs using the 802.11p/WAVE standards are difficult to expect, due to the large investment.

Recently, the pace of Long-Term Evolution (LTE) deployment has accelerated over the world for increasing datahungry smart devices. Widely deployed LTE networks, instead of dedicated ITS infrastructure, can provide trafficcontrol and road-safety services. 3rd-Generation Partnership Project (3GPP) system architecture (SA) WG 1 which defines service requirements for LTE system also completed requirements for vehicular communication in LTE networks [4]. Furthermore, the automotive industry is now embedding LTE clients in vehicles to realize "connected car" with a constant Internet connection for various telematics services, which can be used for safety purposes.

Two types of safety messages are defined in the standard: a Cooperative Awareness Message (CAM) and a Decentralized 
Environmental Notification Message (DENM) [5, 6]. CAMs are periodically broadcasted (in the range of $1-10 \mathrm{~Hz}$ ) to neighboring vehicles to advise them of the senders' direction, speed, and geolocation. DENMs are triggered by emergencies and specific purposes. Vehicles broadcast those safety messages in $\mathrm{V} 2 \mathrm{~V}$ direct communication with a Decentralized Congestion Control (DCC) function to avoid congestion in the designated radio frequency, which is also required in the regulation.

RSU infrastructure using LTE towers has the advantages of preventing transmission collisions by centralized transmission control and covering a large road area with line of sight; contrarily, V2V direct communication can suffer from shadowing by big trucks in the roads. However, CAMs are more of a challenge for LTE RSUs (i.e., LTE base station/eNB) because an LTE eNB has limited capacity to serve periodic CAMs from all vehicles within a cell. A DENM that is less frequently invoked, for an emergency stop or an intersection collision warning, is considered as a major use case for the LTE networks [6]. However, dual-radios of 802.11p/WAVE for CAMs and LTE for DENMs are costly and inefficient.

Standards and literatures clarified that the CAM and DENM should be delivered with the expected service requirement of $100 \mathrm{~ms}$ end-to-end latency $[5,6]$. However, relaying CAMs or DENMs from one vehicle to another is still a challenge for current LTE networks, in terms of limited channel capacity and network architecture. Fortunately, LTE system has several useful features which apply to road safety and traffic control, such as Group Communication System Enablers (GCSE) and Evolved Multimedia Broadcast Multicast Service (eMBMS). Previous studies about vehicular safety communication using LTE networks have investigated scalability and delay problems according to the large number of vehicles or the scheduling mechanisms in eNBs [7-9]. However, a detailed analysis based on the 3GPP LTE standard in order to investigate the feasibility of LTE RSUs has not yet been conducted.

In this paper, we first introduce the useful features of the 3GPP LTE standards for vehicular communications. Then, we investigate their feasibility for CAM and DENM delivery in terms of network overhead and end-to-end delay and finally propose three key ideas to reduce the overhead and delay to satisfy safety requirements. The rest of this paper is organized as follows. In Section 2, we introduce previous works on the LTE system for vehicular communications. In Sections 3 and 4, we overview eMBMS and GCSE as representative LTE features for vehicular communication. We propose an enhanced LTE network model for vehicular safety applications in Section 5 and discuss implementation issue of our idea in Section 6. We evaluate performance of conventional and proposed approaches in Section 7. We discuss remain challenges and conclude in Sections 8 and 9.

\section{Related Works}

Several researches have been conducted on the applicability of LTE cellular technology to vehicular safety communication. Vinel [10] compared the IEEE 802.11p/WAVE and
LTE system in terms of delay and scalability for vehicular safety applications. Mathematical models of the IEEE 802.11p/Carrier Sense Multiple Access (CSMA) and TimeDivision Long-Term Evolution (TD-LTE) were developed to compare their system capacity. According to the analysis results, the LTE system was inadequate to support beaconing messages, such as CAMs, because of its limited capacity. Other existing literatures $[8,9]$ also mentioned that downlink and uplink channel capacities are limited for CAMs. In detail, downlink channels suffer from congestion more in unicast mode than broadcast mode, and the expiration of the CAM deadline (i.e., $100 \mathrm{~ms}$ ) increases exponentially as the number of vehicles exceeds 100. In addition, the CAM delivery success ratio is dependent on the uplink capacity. TD-LTE, with an up to downlink ratio of $1: 9$, shows only $60 \%$ delivery success because of the limited uplink capability. Accordingly, scalability should be enhanced in both up and downlink, for example, by broadcast or multicast instead of unicast and access control for uplink.

Kihl et al. [11] proposed eMBMS (i.e., multicast in downlink) based vehicular communication in the LTE network. The authors assumed a DENM scenario rather than the CAM for V2I communication, since DENM is infrequently generated. Simulation results show that among several scheduling methods, delay-based weight scheduling can satisfy a target delay, $100 \mathrm{~ms}$ of safety applications with at least 50 vehicles. Unfortunately, no results are available regarding situations with more than 50 vehicles, which could be a practical assumption considering the normal macrocell range. Furthermore, a detailed analysis of the latency is missing.

Mangel et al. [12] showed a numerical delay analysis of the Universal Mobile Telecommunications System (UMTS) and the LTE system based on 3GPP standard specifications. They argued that the LTE system could meet the safety message deadline better than UMTS, at least in the random access delay for the uplink transmission if the LTE system deals with 1,500 CAMs per second using the eMBMS in downlink transmission. Although this study opens the possibility of LTE for CAM delivery, it does not show a detailed procedure for vehicular communication in the LTE network. [13, 14] propose the eMBMS based adaptive CAM rate control that can invoke congestion in safety channels, which disturbs emergency messages dissemination in time. In [15], authors derive uplink capacity for CAM dissemination at intersection for varying number of vehicles, where the vehicle broadcasts the CAM through designated radio resource blocks using GPS information. However, detail implementation is not described. [16] describes a detailed architecture of LTE eMBMS for CAM and DEMN dissemination and shows the eMBMS can improve network efficiency and latency when number of vehicles is high enough.

A survey paper [7] introduced existing issues in the LTE system and network deployment for vehicular communications. For the downlink transmission, the authors pointed out that eMBMS causes additional delay due to its session establishment procedures even though it uses fewer radio resources than unicast. In the matter of vehicular network deployment, a back-end server for road safety can reduce the workload of vehicles and eNBs. For example, the server only 
disseminates CAMs or DENMs to geolocationally relevant vehicles since the safety communication range of the vehicles is not likely coincident with the LTE cell area. In addition, an eNB or the server can ignore duplicate messages triggered by the same event or aggregate consecutive messages that are destined for the same vehicle to reduce number of transmissions, which has been explored in ETSI C-ITS under GeoNetworking [17]. Another survey [18] introduces many studies about Heterogeneous Vehicular Network (HetVNET) that integrates cellular networks with Dedicated Short-Range Communications (DSRC). This survey provides comprehension of recent wireless networks techniques for HetVNETs that is still at beginning phase.

For machine type communication (MTC), 3GPP has explored several techniques to support coexistence of the MTC traffic with legacy UE in terms of random access overload [19]. [20, 21] also introduce QoS based access barring approach for different classes of MTC devices in 3GPP LTEA networks. Here RACHs are preallocated or barred dynamically for different MTC classes with different backoff procedures. In [22], authors propose scheduling schemes for uplink channel of LTE for MTC considering QoS such as throughput and allowed delay of each device. [23] proposes load-aware association at overlapped cell area for MTC devices. In [24], device to device (D2D) communication of 3GPP Rel12 is adopted for dedicated broadcast/multicast vehicular safety communications with eMBMS based D2D resource allocation. In [25], authors review various approaches of the D2D communication for vehicular safety communications. They classify those approaches by operator assistance, discovery candidate, QoS, and so forth and compare their performance qualitatively. Recently, [26] enhances the V2V communication for scalability and robustness using LTE D2D and full duplex technologies which improve LTE spectrum reusability and beacon transmission rate for neighbor vehicle awareness compared to previous half-duplex D2D.

\section{Evolved Multimedia Broadcast Multicast Service}

The Evolved Multimedia Broadcast Multicast Service (eMBMS) $[27,28]$ was developed for delivering multimedia broadcast/multicast content to mobile clients, that is, user equipment (UE) in LTE system, which is an effective way to save radio resources for delivering broadcast contents compared to using multiple unicasts. The eMBMS requires several networks' equipment such as Broadcast/Multicast Service Centers (BM-SC), MBMS gateways, Multicell Coordination Entities (MCE), and eNBs, as illustrated in Figure 1(a). The BM-SC is a Broadcast/Multicast Service Center that manages multimedia data along with related information such as the broadcast/multicast area, content, and time and sends them to the MBMS gateways and the MCE. The MCE provides eNBs with control information to schedule their radio resources for the broadcast/multicast data transmission within the eMBMS area. Here the broadcast/multicast radio channel resources are synchronized among multiple cells (Cells 1-4) that participate in concurrent broadcast/multicast transmissions using a single frequency in order to form a large service area as shown in Figure 1(a).

As can be seen in the detailed eMBMS procedures in Figure 1(b), UE first receives System Information Block (SIB) 13 to obtain multicast notifications and the Multicast Control Channel (MCCH) configuration of the corresponding Multimedia Broadcast Single Frequency Network (MBSFN) area [29]. Next, the UE can receive MBSFN Area Configuration through the $\mathrm{MCCH}$ once the UE receives a notification, indicated by an M-RNTI (Multicast Radio Network Temporary Identifier) via the Physical Downlink Control Channel (PDCCH). The MBSFN Area Configuration includes information that identifies which subframes the UE should monitor to obtain the eMBMS data and physical channel characteristics (e.g., Modulation and Coding Scheme (MCS), logical channel Identifier (ID)). With this information, the UE receives broadcast data from the eMBMS channel and decodes them.

Basically, since all broadcast data are delivered through the eMBMS channel, the UE has to know scheduling information of the Multicast Traffic Channel (MTCH) of interest. Such scheduling information can be acquired from the $\mathrm{MCH}$ Scheduling Information (MSI) with a logical channel ID of the MTCH. Then, the UE keeps receiving the MTCH of the logical channel that the UE is interested in. The logical ID is mapped with a Temporary Mobile Group Identity (TMGI) as a session ID in an application layer. Thus, the UE manages both the logical channel ID and the TMGI together after the UE selects a broadcast/multicast session with the TMGI in the application layer.

The above complicated eMBMS procedure is designed for multimedia broadcast/multicast purposes not for realtime bidirectional communications, and it causes a long end-to-end delay. The total delay can be more than $100 \mathrm{~ms}$, which consists of at least $80 \mathrm{~ms}$ for SIB 13, $40 \mathrm{~ms}$ for MSI acquisition, and several more subframes until receiving the actual data, depending on MTCH scheduling. This is far more than the vehicular communication safety requirements, that is, less than $100 \mathrm{~ms}$. In connected mode, an UE could receive data within $100 \mathrm{~ms}$, assuming the UE already knows the MBSFN Area Configuration and the MSI. However, it could be challenge to acquire the configuration information at real time when UE move and they need a new configuration of a changed eMBMS area. To avoid this service interruption, the configuration can be made before the vehicle enters a new area otherwise. If the single eMBMS area covers all roads in wide area, that is, several $\mathrm{km}$, irrelevant safety information (DENM is only required to propagate within $300 \mathrm{~m}[5,6]$.) will be broadcasted over the entire eMBMS area, which wastes radio resources significantly and causes scalability problem.

\section{Group Communication System Enablers (GCSE)}

A Group Communication System Enabler (GCSE) was standardized for group communication such as a mission-critical push to talk (MC-PTT) using unicasts or multicasts in LTE 


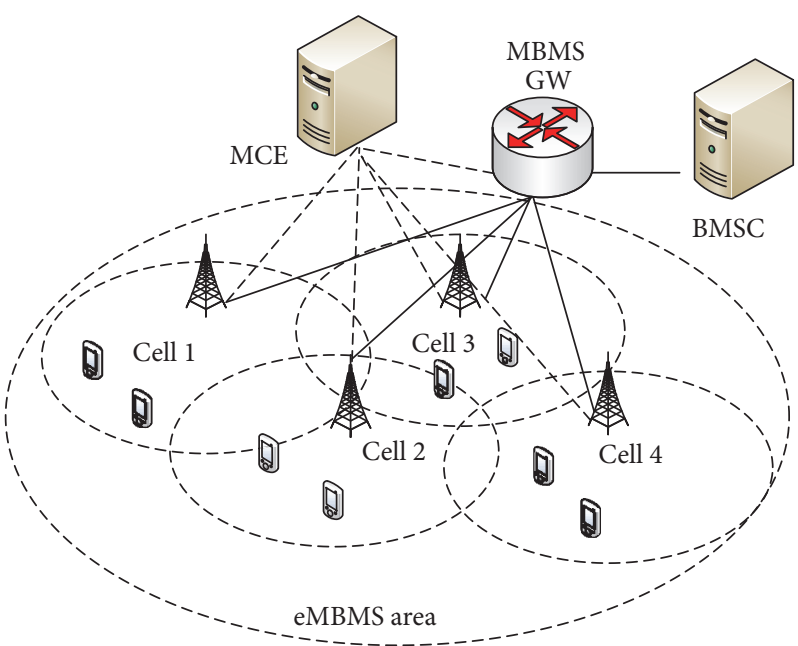

(a) eMBMS architecture

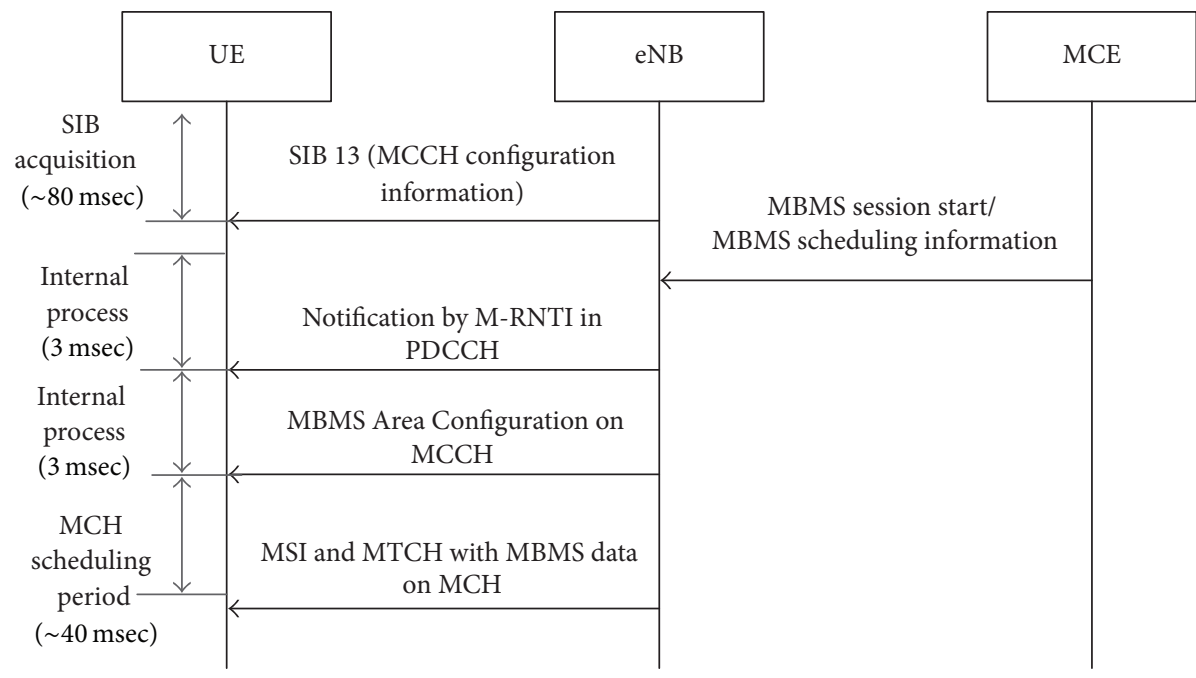

(b) eMBMS procedure

FIGURE 1: eMBMS architecture and multicast data reception procedure.

networks [30]. The GCSE can be applied to vehicular group communication. Figure 2 depicts the GCSE architecture that consists of a GCS application server (AS), the BM-SC, and the MBMS gateway, in addition to an Evolved Packet Core (EPC) network (i.e., eNBs and PDN-GW (P-GW)). The GCS AS manages group members and relays data from one to the others using multiple unicasts or a single multicast. For instance, suppose that UE 1-6 belong to the same group and UE 1 tries to send data to others, UE 2 receives the data by unicast and others receive it by multicast as shown in Figure 2.

For the multicast, the GCS AS requests a Temporary Mobile Group Identity (TMGI) to the BM-SC that manages multicast streaming to multiple eNBs through the MBMSGW and then sends the TMGI to group members using application protocols. The GCS AS has unicast connections to the member UE before changing the unicast connections into a multicast connection. The GCS AS determines an optimal time to switch from multiple unicasts to single multicast and vice versa. The eMBMS for the multicast can consume more radio resources than the multiple unicasts according to number of member UE since the multicast requires designated radio resources periodically regardless of traffic presence. Once the multicast group is established, the GCS AS can activate or deactivate the multicast bearer with Quality of Service (QoS), session start time, MBMS area information, and so forth.

According to [31], a high priority QoS Class Indicator (QCI), even for MC-PTT, has very relaxed requirements: GBR voice (75-100 ms), non-GBR signalling (60 ms), and data $(200 \mathrm{~ms})$ for one-way delay from a gateway to UE. Thus, the round-trip time based on these requirements cannot satisfy the maximum delay for vehicular safety defined in the ITS standardization organization and governments $[5,6]$, since interaction between humans is typically more tolerant than communication between machines. 


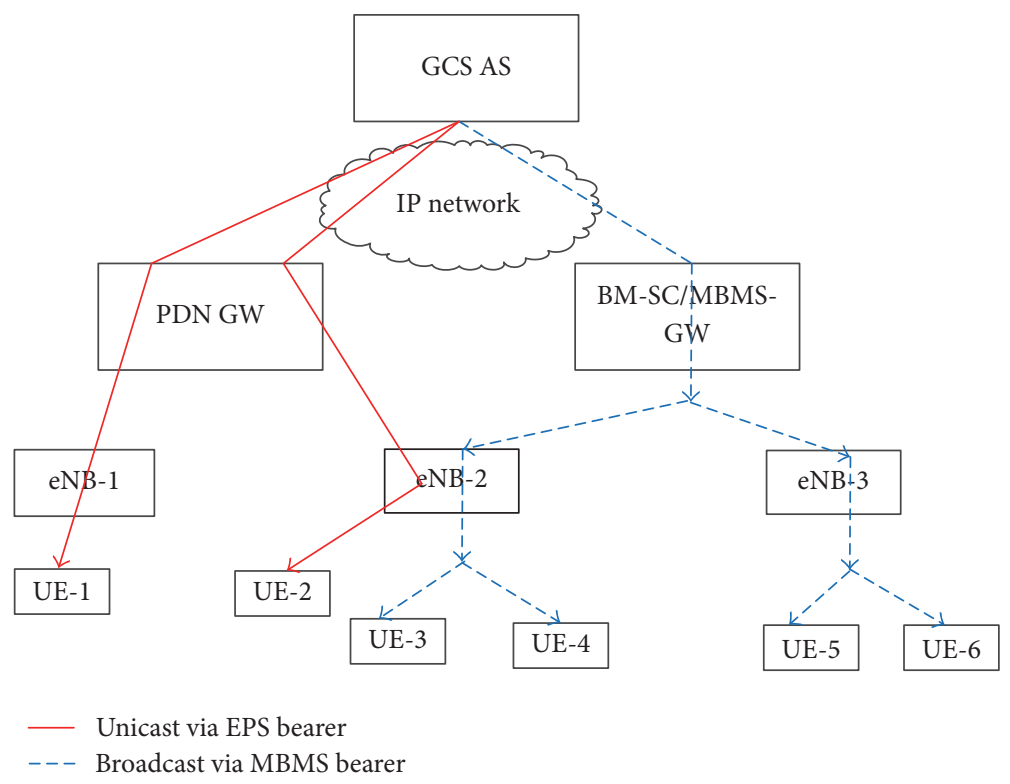

FIGURE 2: GCSE service architecture.

\section{LTE Enhancement for Vehicular Communication}

In previous sections, we overviewed useful features of LTE system for vehicular safety communications. Basically, those features allow vehicle UE to exchange CAMs or DENMs without modification of the current LTE system. However, end-to-end delay requirement for safety messages delivery is guaranteed in the current system.

Three major delay factors in the current LTE communication for vehicular group communications are defined as follows:

(i) Random access (UE-eNB): delay from uplink access and data transmission by idle or connected UE

(ii) Group management (UE-GCS AS): delay from joining or leaving the group with long RTT from eNB to GCS AS since the GCS AS is located outside the EPC

(iii) Downlink configuration (UE-BM-SC): delay from eMBMS configuration information acquisition

In this section, we propose new enhancements for the LTE system to enable delay-critical communication for road safety, which lead to solving above three delay problems. Solutions for each delay term are summarized as below and details of each solution are introduced in following sections:

(i) A persistent uplink channel based on geolocation: to reduce the access delay and resource waste

(ii) A Mobile Edge Cloud (MEC): to reduce the RTT between UE and GCS AS

(iii) A cell-based multicast with geolocation: to reduce configuration delay for multicast stream reception

5.1. Geolocation-Based Persistent Uplink Channel. For the initial uplink transmission, UE should first perform a random access in order to maintain orthogonality among uplink transmissions from many UE. An eNB synchronizes the arrival times of the uplink signals from the UE by shifting the transmission time according to the UE's locations.

As shown in the detailed random access procedure in Figure 3, UE first sends a randomly chosen preamble to eNB through a random access channel (RACH) and waits for a random access response (RAR). The RAR contains the preamble index, uplink timing adjustment, and uplink resource assignment information for the UE. The UE sends a connection request using the assigned resource with the UE identity (ID) if the received preamble index is the same as the sent preamble. The UE ID is used for contention resolution, in case more than two UE setups coincidentally send the same preamble. The eNB returns the ID to the UE with a new Cell Radio Network Temporary Identifier (C-RNTI) as a radio connection ID in the connection setup message. Next, the UE sends the C-RNTI and a Buffer Status Report (BSR) message in the connection-complete message and receives an uplink grant with the C-RNTI for actual data.

The random access procedure takes a total of $67 \mathrm{~ms}$ on average, as calculated in Table 1 and Figure 3. However, the initial access latency increases exponentially as attempts fail. If eNB uses backoff indication to control the access attempts of many UE setups, it can take more than several seconds because the maximum backoff time is $960 \mathrm{~ms}$, and up to 200 random access retries are allowed in the standard. Only two retries with several ms backoffs could defeat our latency goal, even without considering additional hybrid automatic-repeat request (HARQ) delays from retransmissions.

In [7], authors argue that vehicle clients can always be connected to reduce the initial access delays. The connected UE instead need a scheduling request (SR) procedure for the uplink grant, as shown in Figure 4. For the SR, a periodic Physical Uplink Control Channel (PUCCH) is assigned for 


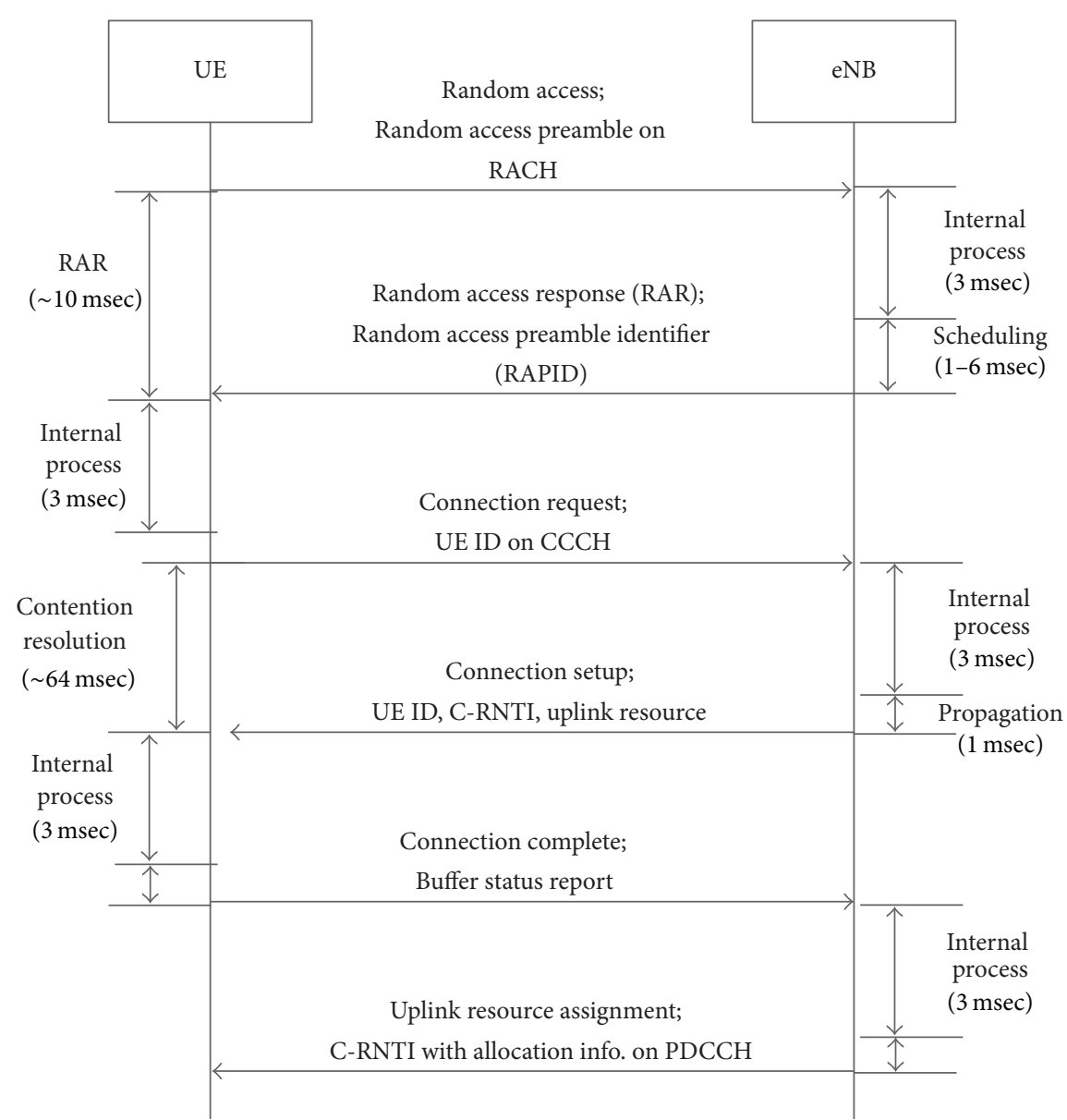

FIGURE 3: Random access procedure and delay of each step.

TABLE 1: Random access delay analysis.

\begin{tabular}{|c|c|c|}
\hline Procedure & Detail & Total average delay \\
\hline \multirow{3}{*}{ Random access (RA) } & RACH subframe $[1,20 \mathrm{~ms}]$ & \multirow{3}{*}{$19 \mathrm{~ms}$} \\
\hline & $\operatorname{RAR}$ window $[1,10 \mathrm{~ms}]$ & \\
\hline & RAR process $[4 \mathrm{~ms}]$ & \\
\hline \multirow{3}{*}{ Contention resolution (CR) } & Connection request $[4 \mathrm{~ms}]$ & \multirow{3}{*}{$40 \mathrm{~ms}$} \\
\hline & Connection setup $[1,64 \mathrm{~ms}]$ & \\
\hline & Connection setup process [ $4 \mathrm{~ms}]$ & \\
\hline \multirow{2}{*}{ Uplink resource allocation (UL) } & Buffer status report [4 ms] & \multirow{2}{*}{$8 \mathrm{~ms}$} \\
\hline & Uplink grant process [4 ms] & \\
\hline
\end{tabular}

each UE; otherwise, the UE must perform a random access procedure. The SR period depends on the network load; the period tends to be longer if eNB has many connected UE to schedule on the limited PUCCH for the SR.

As a detail of the SR procedure, the UE sends the SR via the PUCCH, and the eNB assigns an uplink grant for the BSR. The UE reports the current buffer status using the BSR and receives an uplink grant for data. The two request/response procedures for BSR and data take $8 \mathrm{~ms}$ each. On average, a total of 21 to $26 \mathrm{~ms}$ is spent transmitting safety messages, assuming the SR period is around $10 \mathrm{~ms}$. It takes longer if the BSR or data transmission fails (e.g., 8 additional ms for each retransmission). For instance, if two retransmissions occur, a total of $42 \mathrm{~ms}$ is necessary.

The delay of the connected UE allows safety messages to be delivered within the deadline. However, connecting all UE with periodic PUCCH resources for the SR is inefficient in terms of scalability and resource wastes; the delay will increase according to the number of connected UE setups. Supposing that the PUCCH is allocated around 5\% of the $5 \mathrm{MHz}$ uplink resources, 17 UE setups can have the SR every Transmission Time Interval (TTI); each radio block (RB) is normally multiplexed by 17 UE setups for PUCCH format 1 of the SR. Thus, a total of 170 connected UE setups are supported 


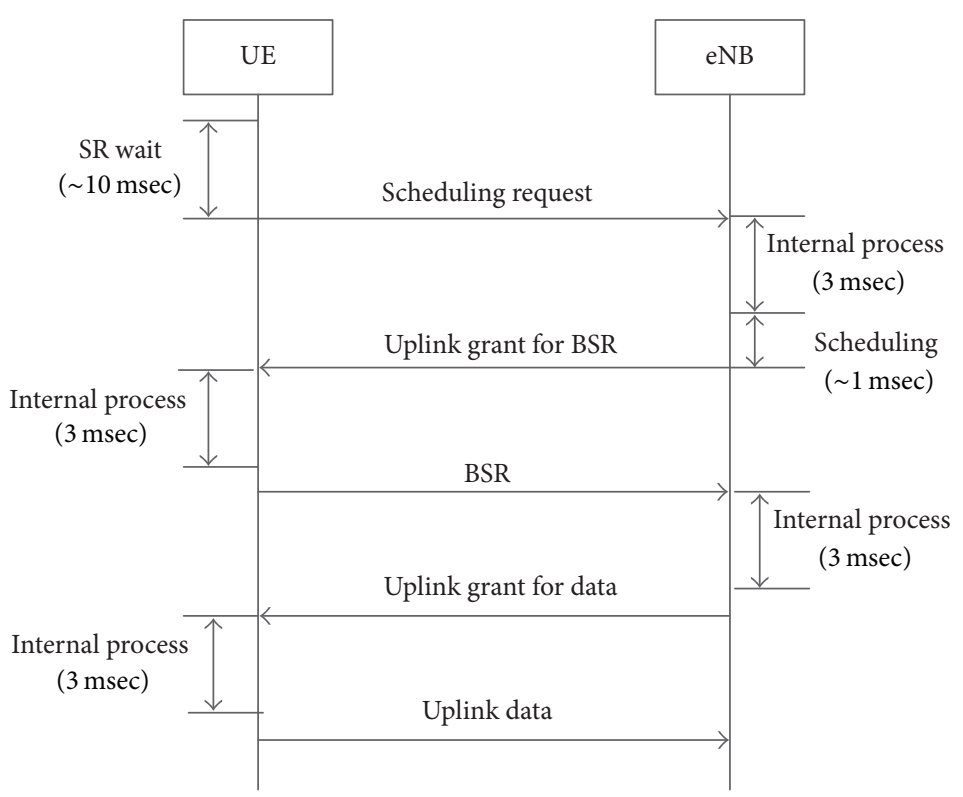

FIGURE 4: Scheduling request procedure and delay of each step.

with a $10 \mathrm{~ms}$ SR period. However, the eNB may need to extend the SR period or increase the PUCCH resources, since the 3GPP LTE standard requires a minimum of 300 UE setups without Discontinuous Reception (DRX), according to [32], and the conventional UEs such as handheld devices may coexist. For example, 600 connected UEs (e.g., 300 vehicles and 300 legacy UEs) lead to an increase in the SR period of about $50 \mathrm{~ms}$, which is still valid, but can be a challenging value if transmission failure occurs. Furthermore, the periodic SR resource of PUCCH for each UE is wasted if there is no data to send. Considering limited resource of the PUCCH, an alternative method to simultaneously reduce the delay and overhead should be considered. Always connected vehicles also cause significant overhead to eNB due to frequent handover procedures.

We propose a geolocation-based common persistent channel for idle or connected vehicles in LTE networks as described in Figure 5. The eNB assigns uplink resources periodically for common persistent channels in which many vehicles send safety messages directly to the eNB by competition. This common uplink (CUL) channels allow skipping the SR and BSR procedures and reduce uplink resource waste by sharing the uplink resource with others.

Within the common channel, there is unfortunately no mechanism to avoid collision completely, like Wi-Fis listenbefore-talk. In order to reduce collisions and resource waste, therefore the persistent common channels can be assigned according to geographical location, that is, a road segments $1-4$, as can be seen in Figure 5. The eNB can schedule uplink channel resources dynamically based on the vehicle density per segment. In the figure, number of vehicles is different to the road segments. Accordingly, the eNB can assign more common channels to segment 1 rather than others. In practice, the eNB can configure the common channel patterns using period and offset values based on the road density.
Above approach has twofold critical problems; first, random access procedure is still necessary for idle UE. Secondly, CUL channels are also can be wasted. Thus, additional geolocation-based random access is conducted for idle state UE and collision avoidance before CUL channels. Figure 6 shows a detailed procedure of our proposed scheme. UE selects a preamble and sends it through a random access channel (RACH) (noted as R1-R4 in Figure 5) that the eNB assigns periodically for each road segment. Only UE which receives the RAR from the eNB can send prepared packets of safety messages through the persistent channel of the road segment (noted as $\mathrm{Cl}-\mathrm{C} 4$ in Figure 5). The RAR PDU includes multiple RARs for UE which is limited by a transmission block (TB) size. Currently, only a few UE setups, for example, less than 5 UE setups, are addressed in the same RAR PDU. However, vehicular communication can generate more simultaneous RA procedures. For our approach, the eNB has to assign enough resources for the RAR PDU before the CUL channel. For example, suppose maximum TB size for $5 \mathrm{MHz}$ (25 PRBs) is 1096 bits with MCS $=2$ and 2 PDCCH symbols, then about 19 RARs can be delivered in each subframe (each RAR is 7 bytes including a header). Thus, the eNB can send more than 50 RARs using 3 subframes before the CUL channel subframe. However, UE may not receive the RAR due to RAR overhead in RA explosion. In such situation, a current LTE system defers RA attempts for a while using backoff or blocks RA from UE in a certain category using system information. Similarly, our approach also defers to perform RA until only several consecutive RACH opportunities due to delaysensitive vehicular communication.

Finally, our approach would take around $10-15 \mathrm{~ms}$ from the initial access to sending a safety message, depending on persistent channel schedule, and is also able to apply to idle UE. Furthermore, the eNB can reassign uplink resources 


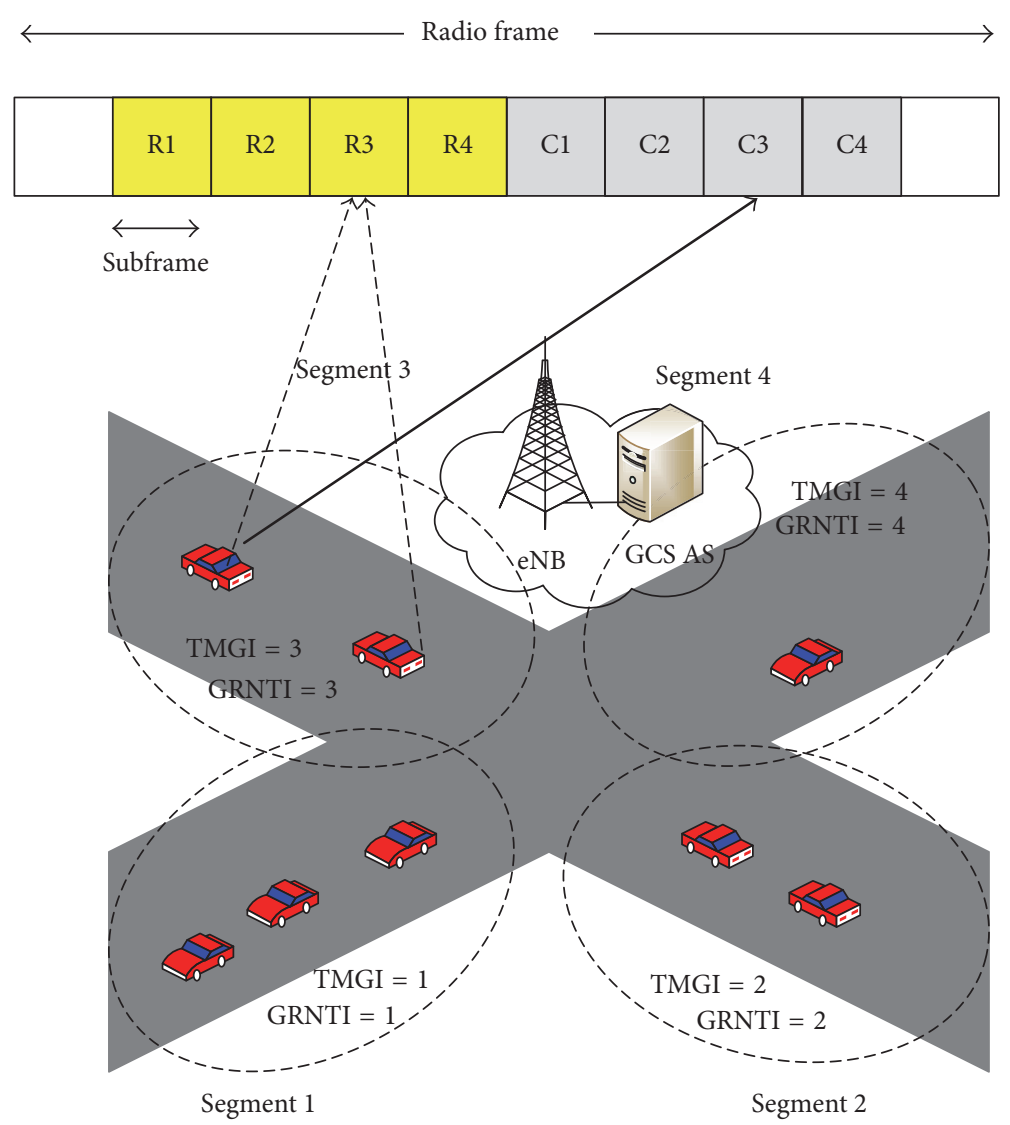

FIGURE 5: Geolocation-based uplink and downlink channel assignment model.

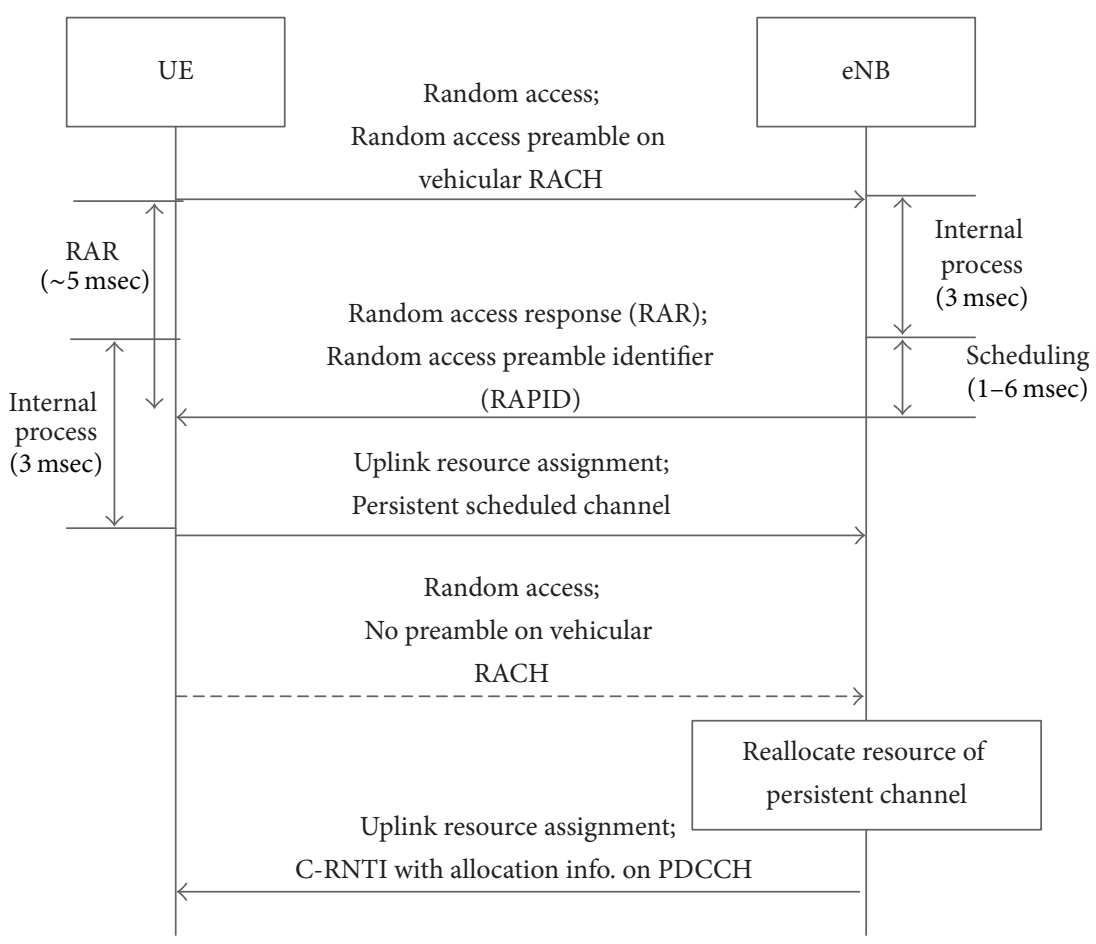

FIGURE 6: Our proposed approach for safety message uplink transmissions. 
reserved for the persistent uplink channel to other uplink channels if no preamble appears in the corresponding RACH, which can prevent uplink resource waste. For instance, if there is no preamble detected in $\mathrm{R} 2$ in the figure, the common channel C2 can be reused for uplink transmissions for other UE setups.

Our approach requires precise geolocation information to allocate radio resources for fine road segments, which is assumed to get basically a precise GPS receiver, but many state-of-the-art localization techniques such as crowdsourced $\mathrm{V} 2 \mathrm{~V}$ and dead reckoning can be considered additionally. Further enhanced localization techniques can improve flexibility in resource scheduling.

\subsection{Group Communication Application Server on Mobile Edge} Computing. When a vehicle sends a DENM message to the GCS AS out of the EPC network, the message must travel through the EPC network and external networks like the Internet to reach the GCS AS. Further, the message should return to the LTE network to be distributed to member vehicles using a multicast. We can shorten the long safety message route by locating the GCS AS close to the UE, as shown in Figure 5. We can remove the entire backhaul delay, more than $20-30 \mathrm{~ms}$, if we place the GCS AS inside eNB. Recently, Mobile Edge Computing (MEC), the ETSI standard working group, has defined programmable APIs for computing platforms to be located inside the eNB or near the cell site. The MEC is applicable to vehicular communication since it occurs within a specific traffic area (e.g., $300 \mathrm{~m}$ for the DENM) that is usually smaller than LTE cell area (e.g., $500 \mathrm{~m}-1 \mathrm{~km}$ ). However, the safety messages from the cell-edge area may need to be relayed to neighboring eNBs if every eNB has an individual GCS AS inside. A hierarchical GCS AS architecture can be considered for such cases.

In current LTE networks, all bearers have always to be terminated at the gateway. Thus, the gateway controls the route of the group communication bearer to the GCS AS, based on policies given by a policy server once the application of vehicular group communication creates a group, using protocols like SIP, HTTP, and so forth.

However, in the proposed architecture, eNBs should deal with traffic steering and QoS guarantees for vehicular safety communication. For this, LTE specification has to be changed to terminate UE bearers at the eNB instead of the gateway for the MEC-based applications/ services like GCS AS. Alternatively, software define networking (SDN) technology can be applied on top of the legacy LTE network [33], where the MEC provides northbound APIs for the traffic steering to establish directly an end-to-end path for a bearer on top of legacy LTE nodes using switch control protocols like OpenFlow [34-36]. Accordingly, radio bearers are routed to application servers in the MEC by IP-based routing rather than GTP-based tunneling. Recently, many researches about 5G explore the traffic steering techniques using the SDN to support handover and edge cloud computing in a flat architecture of wireless access and core networks.
5.3. Cell-Based Multicast with Geodata. The eMBMS is effective for improving downlink scalability but inappropriate for delay-critical machine-to-machine (M2M) group communication, such as vehicular safety communication. In human group communication, the group-call setup delay for mission-critical purposes requires less than $300 \mathrm{~ms}$. Vehicular communication is more dynamic than human group communication in terms of joining or leaving the group. Thus, multicast session acquisition for group communication for vehicles should be performed faster than in the current approach. Thus, the eMBMS configuration and TMGI acquisition delay should be reduced.

Simple mapping of the TMGI to a road segment, as in Figure 5, can remove the current lengthy group establishment procedure that allocates the TMGI to a certain group in the eMBMS system. Vehicles can derive the group TMGI of the corresponding road segment based on geolocation information (i.e., GPS and map data). So our approach does not need the group join and leave procedures caused by mobility. The mapping information is given by an eNB using radio resource control (RRC) messages that are faster than SIB, when the vehicles enter the LTE cell area initially or by handover. Although the group establishment delay can be ignored, there is still a delay from acquiring the eMBMS configuration information. Using eMBMS for vehicular communications can be costly according to amount of safety data. Switching between multiple unicasts and multicasts using the eMBMS based on the data also causes signalling overhead in EPC networks.

Multicast using a Physical Downlink Shared Channel (PDSCH) (i.e., cell-based multicast) can be a more flexible and efficient alternative for vehicular group communication than the eMBMS because vehicular group communication area is not wide unlike conventional eMBMS service area; a single LTE eNB can cover the communication area. 3GPP studied recently this single-cell point-to-multipoint transmission [37]. The eNB assigns dynamically multicast downlink resources only when necessary, instead of the fixed downlink resources for the eMBMS. The eNB sends multicast data via normal PDSCH with a group RNTI (G-RNTI), a new RNTI for the group communication. Once the UEs receive the PDCCH, they first descramble the PDCCH area with the C-RNTI as a blind search in order to find the downlink channel assignment (i.e., time/frequency resources) and the MCS in the PDSCH for unicast. After that, they repeat the procedure with the G-RNTI to look for a multicast channel assignment. After then, UE decode PDSCH with those channel assignment information to obtain unicast and multicast packets. Consequently, this cell-based multicast can save downlink resources and remove the prerequisite procedures to receive group data, such as the eMBMS configuration and $\mathrm{MCH}$ Scheduling Information reception.

The G-RNTI can be assigned to each road segment along with the TMGI as shown in Figure 5. A safety message broadcast area might not be exactly the same as the road segment. For instance, highway road segments are continuous compared to those in the Manhattan grid. The broadcast area can be parts of two segments in such situations. In addition, 


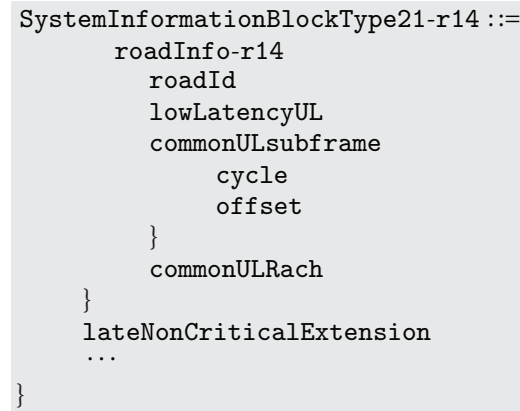

SEQUENCE \{

SEQUENCE \{

INTEGER ( $0 \cdots 549755813887)$

SEQUENCE \{

SEQUENCE \{

INTEGER (0 ‥5 549755813887), -- Need OR

INTEGER (0 . . 549755813887), -- Need OR

RACH-ConfigDedicated

OCTET STRING

OPTIONAL ,

FIGURE 9: System Information Block for common uplink and RACH resource information.

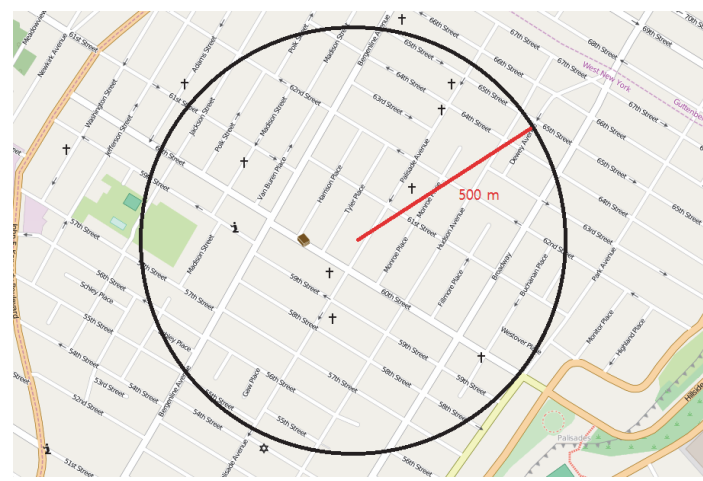

(a) Manhattan

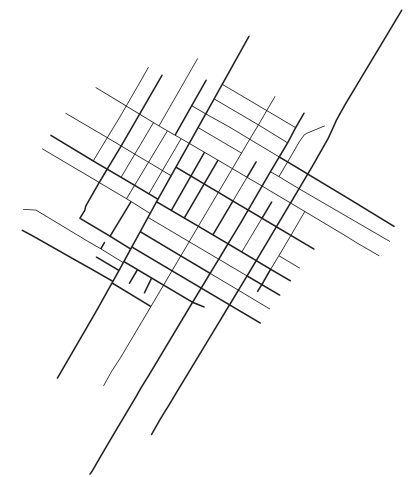

(b) Tiger map

FIGURE 10: Manhattan grid covered by a single macrocell.

derive the key using a network key in a mobility management entity (MME), which causes network traffic between the eNB and MME and key acquisition latency. To avoid this issue, long DRX that has been discussed for MTC can be applied to keep the UE awake [39]. Also, the eNB can keep the context of idle UE to reduce trials to query the security key. Security still has more challenges especially for the idle mode vehicles. We will revisit this issue in future work.

\section{Evaluation}

In this section, we investigate LTE assisted DENM and CAM vehicular communications with simulation and numerical analysis and discuss their feasibility.

First, we evaluate one of DENM applications, Intersection Collision Risk Warning (ICRW) in a single LTE cell under realistic vehicular traffic. The DENM occurs rarely according to traffic situation compared to the CAM. To estimate number of vehicles near intersection for the ICRW, we first conduct preliminary experiment using traffic simulator, Simulation of Urban Mobility (SUMO) [40]. We captured realistic road environment at Manhattan, New York, using OpenStreetMap [41] as shown in Figure 10(a). Figure 10(b) shows a tiger map of the Manhattan area covered by a single macro eNB with $500 \mathrm{~m}$ radius. There are almost 180 road segments with different number of lanes and 100 intersections. Here we count the number of candidate vehicles for the ICRW with varying multiple random trips where multiple vehicle flows (i.e., car follower model) move from one to another road segment in the map.

Figure 11(a) shows average number of vehicles within $5 \mathrm{~m}$ from an intersection (alarm zone) with varying traffic generation durations. According to the duration of traffic generation, the number of vehicles number in the zone is slightly different but comparable. For example, total 24 vehicles are shown in $500 \mathrm{~s}$ while 15 vehicles are detected in $100 \mathrm{~s}$.

In contrast, arrival rate (denoted by $r$ ) of the vehicle flows affects vehicle density notably compared to the generation duration and number of entering vehicle flows (denoted by variable $e$ ), 15 vehicles in $r=1$, but more than 50 vehicles in $r=0.3$ as shown in Figure 11(b) where each vehicle arrives every 1 or $0.3 \mathrm{~s}$ and average 5 vehicle flows are generated, simultaneously. Additionally, the vehicle density highly depends on the size of alarm zone as shown in Figure 11(c); the zone size increases the density more aggressively especially in higher arrival rate (80 vehicles at $10 \mathrm{~m}$ to 140 vehicles at $30 \mathrm{~m}$ with $r=0.3$ ).

As a consequence, vehicle density is related to arrival rate of vehicle flows and vehicle speed. Considering average vehicle speed in the Manhattan (about $9 \mathrm{~m} / \mathrm{s}(32 \mathrm{~km} / \mathrm{h})$ ), the arrival rate might be about $r=0.3$ with either $e=5$ or $e=10$ as shown in Figure 12. Suppose that alarm zone is set as $20 \mathrm{~m}$ (a second distance by maximum speed $60 \mathrm{~km} / \mathrm{h}$ ), about 120 


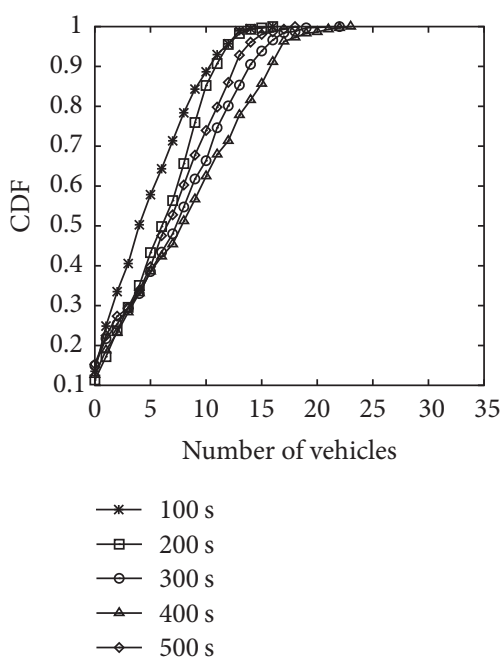

(a) Flow generation durations

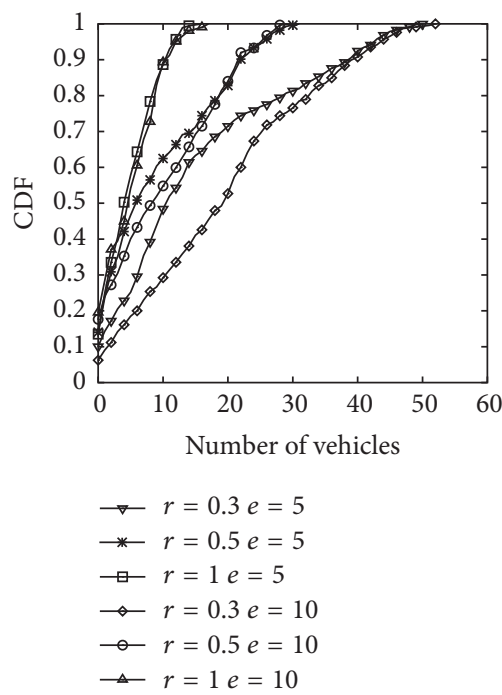

(b) Arrival rate

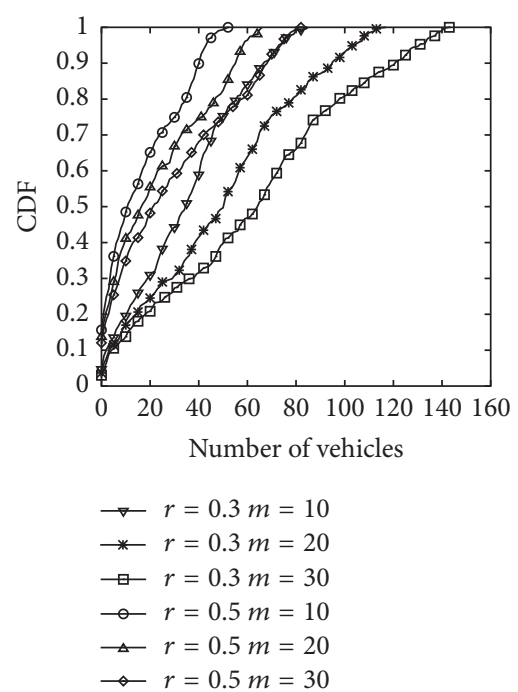

(c) Distance from intersection

FIGURE 11: CDF of vehicle density near intersection area.

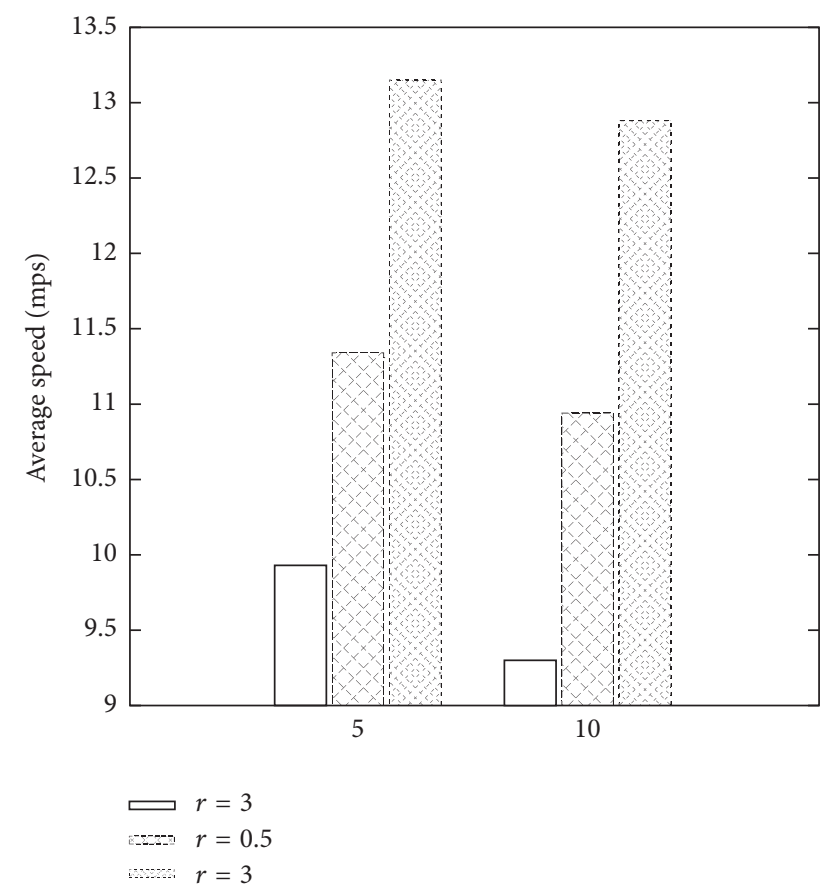

FIGURE 12: Average speed of vehicles in road segment.

DENMs might be necessary for average Manhattan vehicle flow (cf. 140 DENMs for $30 \mathrm{~m}$ ).

In the above example, the eNB should provide $120 \mathrm{RACH}$ opportunities for intersection alarm every second, which does not cause significant overhead in the LTE system since only a couple of RACH opportunities are needed in a single radio frame. In other words, ICRW messages can be delivered probably without collision if the macro eNB assigns only one or two designated RACH or uplink RBs every radio frame for each road segment. Although more RACHs are necessary for more intersections to avoid collision completely, the DENM can be supported normally due to its low overhead.

Unlike an event-triggered message such as the ICRW, CAMs or periodic DENMs dissemination is typically more challenging due to frequency of periodic broadcast and number of vehicles sending messages. For numerical analysis of the CAMs, we establish a simple model that copes with access in RACH or CUL channels with varying arrival rate of the safety messages. If vehicle $i$ does not have queued messages with arrival rate of periodic safety messages, CAMs, $\lambda$, where the message arrivals are exponentially distributed, it can have a new message to send at time $t$ with a probability, $p_{t, 0}$, which is actually a probability of a packet arrival within a unit duration time window (i.e., $\lambda$ is average number of CAMs per LTE subframe).

$$
p_{t, 0}=1-e^{-\lambda}
$$

Some vehicles have safety messages in the queue for retransmission if they fail last time. Backoff window, $\omega$ for random access can be configured to avoid collisions from concurrent transmission because safety messages can be generated simultaneously by multiple vehicles. In this situation, all vehicles with the backlog will send messages at the time $t$, with transmission probability of $p_{t, 1}$.

$$
p_{t, 1}=\frac{2}{\omega+1} \text {. }
$$

At the common uplink data channel, collision probability of vehicle $i$ can be

$$
p_{c, i}=1-\prod_{i \neq j}\left(1-p_{t, 1 \mid 0, j}\right),
$$

where $p_{t, 1 \mid 0, j}$ is a conditional probability of the $p_{t, 1}$ under $p_{t, 0}$, which indicates a transmission probability of node $j$ which has queued packets. 
Even though simultaneous preamble transmissions occur, orthogonal random preambles can be decodable in eNB. Available preambles are different to eNB status; there might be fewer preambles remained for the initial access if the eNB reserves many preambles for designated uplink transmissions. If $r$ preambles are available for the initial random access for vehicles, $n$, which information is broadcasted in SIB2 message, a probability to select duplicate preambles, $p_{r}$, is

$$
p_{r}= \begin{cases}\frac{r !(r-1) !}{(r-n) !(r+n-1) !} & \text { if } r \geq n \\ 1 & \text { if } r<n .\end{cases}
$$

Thus, successful access probability in RACHs $p_{s}$ is derived by failure case in which same preamble is chosen by multiple UE setups which are willing to send the preamble in next RACH subframe.

$$
p_{s}=1-p_{c, i} p_{r} .
$$

To figure out feasibility of our approach, several different approaches to reduce delay from uplink data transmission are compared. Expected delay of each approach is different as below. Delay values $T_{d}$ of each step for an uplink transmission are shown in Table 1. Actual data transmission delay through the uplink data channel is ignored assuming requested grant is fully accepted by eNB. Additionally, $T_{\mathrm{UL}_{P}}$ is considered to investigate delay impact on transmission in a CUL data channel.

(1) Legacy uplink access delay,

$$
T_{d}=T_{\mathrm{RA}}+T_{\mathrm{CR}}+T_{\mathrm{UL}}
$$

If UE fails to acquire uplink grant due to collisions, it has to spend more time of $T_{\mathrm{RA}}+T_{\mathrm{CR}}$ by repeating these procedures. For simplicity, CR failure is not handled in this analysis.

(2) Data transmission in a CUL data channel in this approach, UE send data without initial RA procedure because they are supposed to have connections to eNBs. Thus, transmission failure occurs only by the collision probability.

$$
T_{d}=T_{\mathrm{UL}_{P}} .
$$

This scheme can reduce delay by skipping procedures of $T_{\mathrm{RA}}+T_{\mathrm{CR}}$ for a data transmission, and a part of $T_{\mathrm{UL}}$ can be also omitted because CUL channels are periodically scheduled by eNB. Instead, $T_{\mathrm{UL}_{p}}$ is added since $T_{d}$ depends on eNB scheduling for the CUL.

(3) Random access with a CUL, this approach is also based on the CUL like previous one, but it needs initial RA procedure in advance in order to reduce transmission collisions in the CUL and is applicable to idle UE.

$$
T_{d}=T_{\mathrm{RA}}+T_{\mathrm{UL}_{p}} .
$$

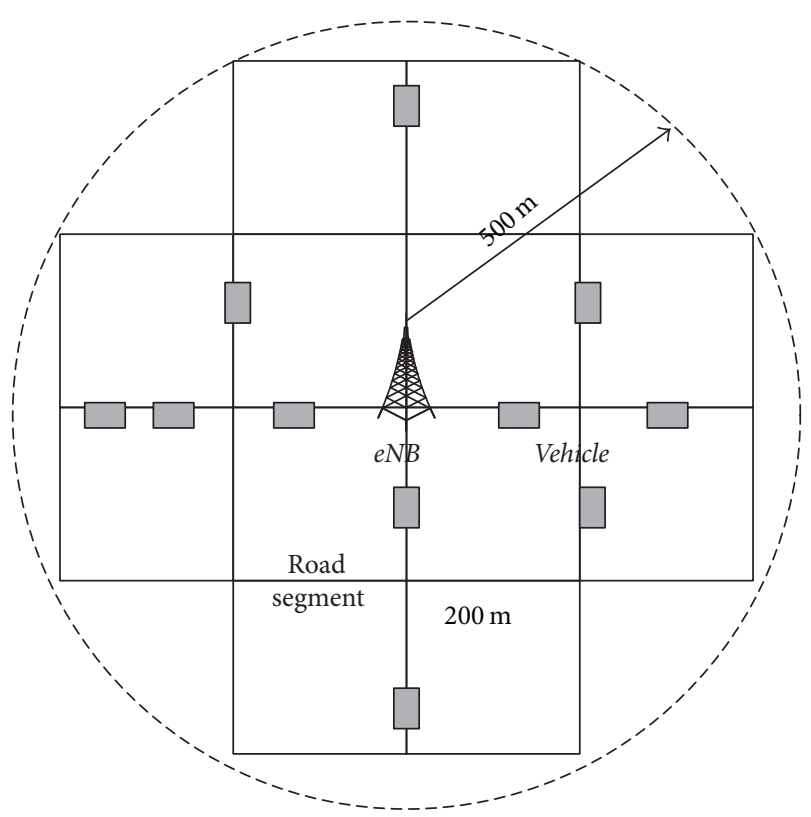

FIGURE 13: LTE network and road topology for evaluation.

(4) Geolocational road based random access (GeoRA) for a CUL, this is our proposed approach that extends previous CUL to RA using geolocation road information. Expected delay is same as the previous RA with the CUL approach in each road segment. However, $T_{\mathrm{RA}}$ is longer than previous one by times of number of road segments because vehicles as UE are distributed over multiple RACHs according to road segments. In other words, the RACH period of our scheme is longer than the previous approach.

$$
T_{d}=T_{\mathrm{RA}}+T_{\mathrm{UL}_{P}} .
$$

Using (1)-(5) and expected delay of each approach, we simulate uplink transmissions for safety messages in a simple one-cell LTE network with varying number of UE and RACH resources. Figure 13 shows a simulation road topology that consists of 30 road segments of $200 \mathrm{~m}$ within the urban LTE cell that covers $500 \mathrm{~m}$ radius range and total 300 vehicles that are distributed uniformly over the road segments and sending safety messages to each other. Herein we assume 16 dedicated preambles are assigned for vehicular communication.

Figure 14(e) shows expected delay to send a single safety message to the eNB with varying number of vehicles. Here we assume that a RACH and a persistent common uplink channel are assigned, respectively, every $10 \mathrm{~ms}$, that is, one subframe per a radio frame. According to the arrival rate of the safety messages, average delay exponentially increases in most of approaches, Figures 16(a)-16(d). In the figure, $\lambda=$ 0.01 means that 10 CAMs are sent per second, and $\lambda=0.001$ is a 1 CAM per second. For the CAM delivery, $\lambda=0.01$, all of approaches do not satisfy deadline, $100 \mathrm{~ms}$ for near $300 \mathrm{UE}$ setups. But lower arrival rate of safety messages (i.e., less than 0.001) like DENMs can be dealt with by most of approaches except GeoRA. In the GeoRA, a period of a dedicated RACH 


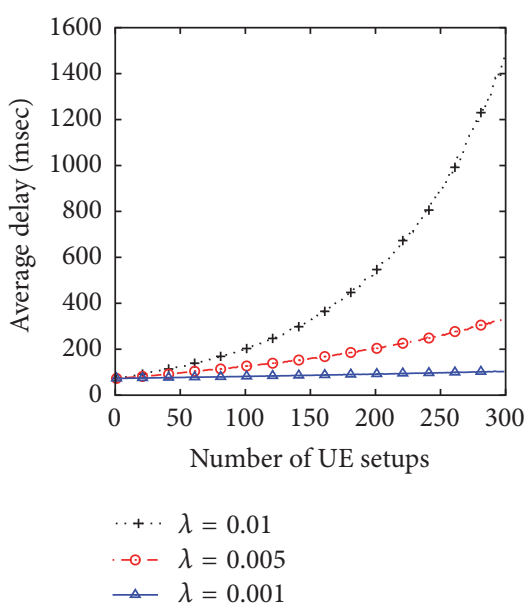

(a) Legacy

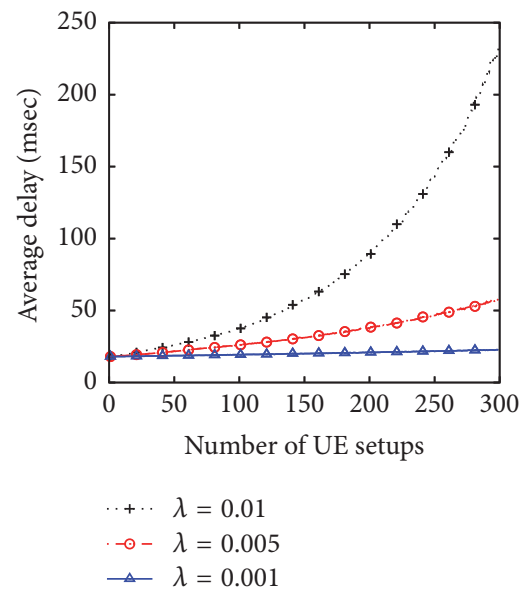

(b) CUL channel

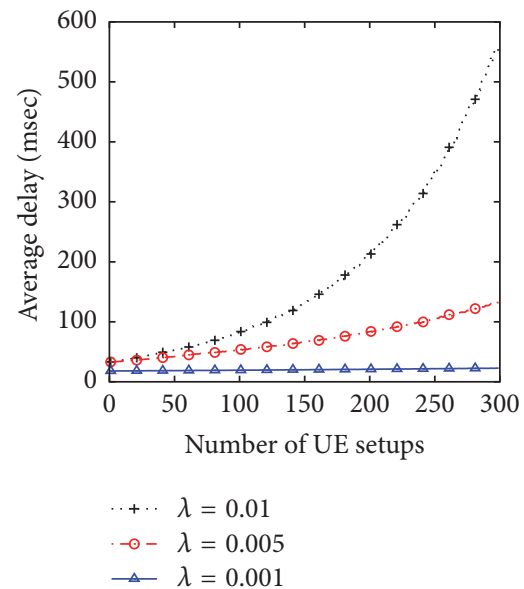

(c) CUL with RA

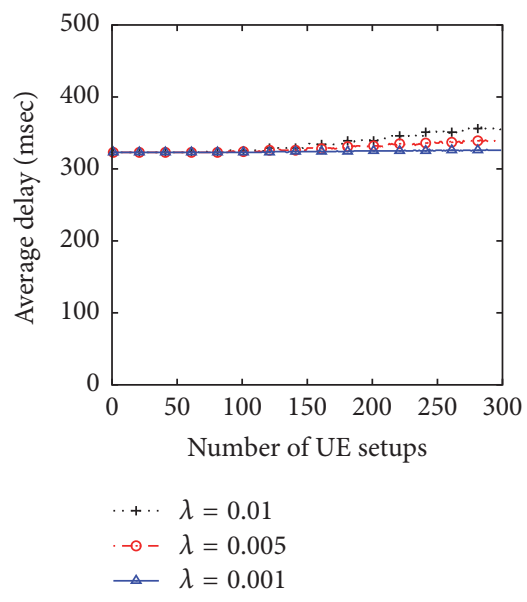

(d) Geolocation road based RA (GeoRA)

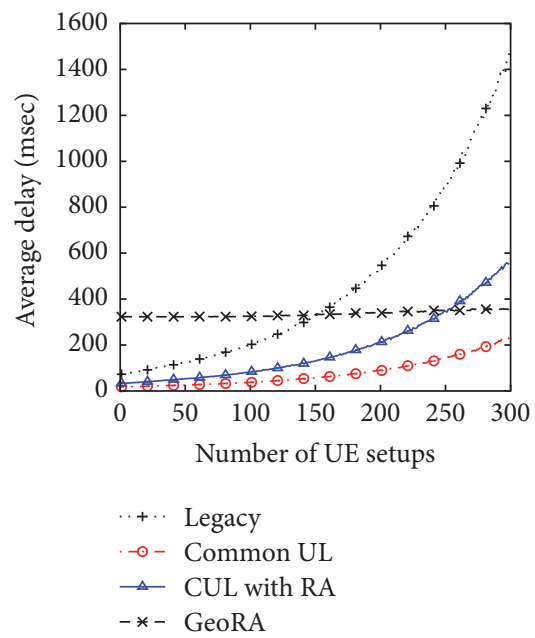

(e) Delay comparison with $\lambda=0.01$

FIGURE 14: Average delay with varying arrival rate of safety messages.

assigned to each road can be $10 \mathrm{~ms} \times 30$ since the $\mathrm{RACH}$ period is multiplied by number of road segments. Figure 14(e) shows comparison of the four approaches with $\lambda=0.01$. CUL channel for data transmission achieves better than others since delay expense from collisions is less than others.

Figure 15 illustrates delay performance of the CUL with different CUL periods (i.e., 10, 5 and $1 \mathrm{~ms}$ ). Frequency of CUL allocation affects average delay as shown in Figure 15(a); average delay increases as the CUL period increases. For the CAM, however, every subframe should have the CUL for more than $300 \mathrm{UE}$ setups. In $5 \mathrm{MHz}$ bandwidth (i.e., 25 physical resource blocks (PRB)), overhead can be near 25\% since 6 PRB is enough for the safety messages with lowest modulation, BPSK (2 kbit message size according to $[42,43]$ ). Additionally, almost 20 times CUL waste could occur for a single safety message transmission in case of the $300 \mathrm{UE}$ setups as shown in Figure 15(b). Random access can reduce such wastes of uplink resources by selecting a transmitter and rescheduling an unused CUL resources to other UE setups. Delay from the random access can be reduced by increasing number of RACHs as follows.
Figure 16 depicts average delay with varying $\mathrm{RACH}$ opportunities for the safety transmission with $\lambda=0.01$. In LTE standard, RACH can be assigned once every radio frame $(\mathrm{RACH}=10 \mathrm{~ms})$, a half of a radio frame $(\mathrm{RACH}=5 \mathrm{~ms})$, even or odd subframe (RACH $=2 \mathrm{~ms}$ ), and so forth. Most of cases except the CUL shows that delay decreases with more RACH opportunities; the CUL approach does not use RACHs. However, deadline requirement of safety purpose cannot be satisfied except the GeoRA with RACHs in every the other subframe. In Figure 16(e), delay comparison with $\mathrm{RACH}=2$ notes that the CUL and the CUL with RA outperform than GeoRA until around 150 UE setups because a longer RACH period of the GeoRA takes probably a large part of total delay in lower collision situation. In consequence, GeoRA can adjust RACH opportunities to improve performance by allocating more RACHs on dense road segments or simply grouping road segments dynamically according to the total number of vehicles.

Figure 17 depicts average delay with varying group size of road segments with every $5 \mathrm{~ms} \mathrm{RACH}$. The group size is a number of road segments which are assigned for each RACH. 


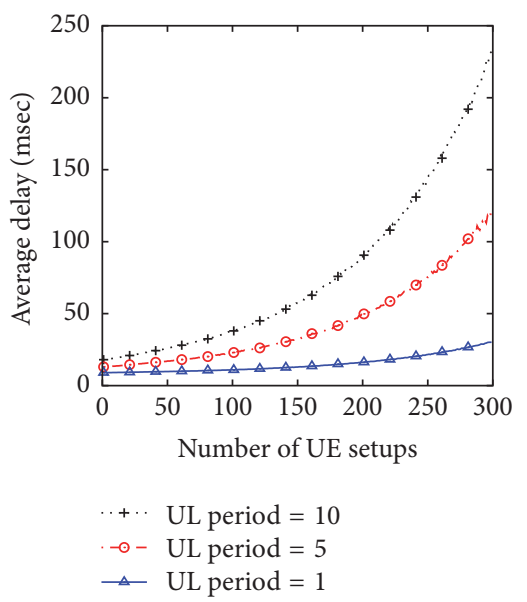

(a) CUL with varying period

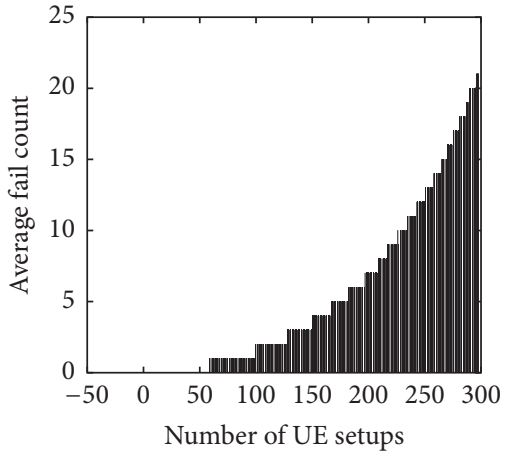

(b) CUL with RA

FIGURE 15: Common uplink data channel access performance.

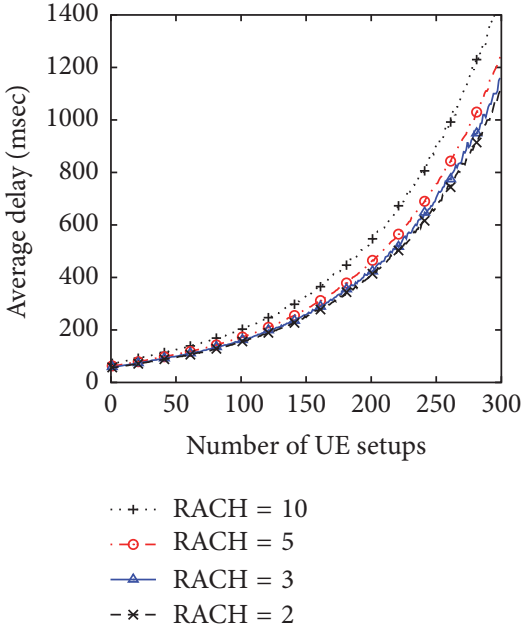

(a) Legacy

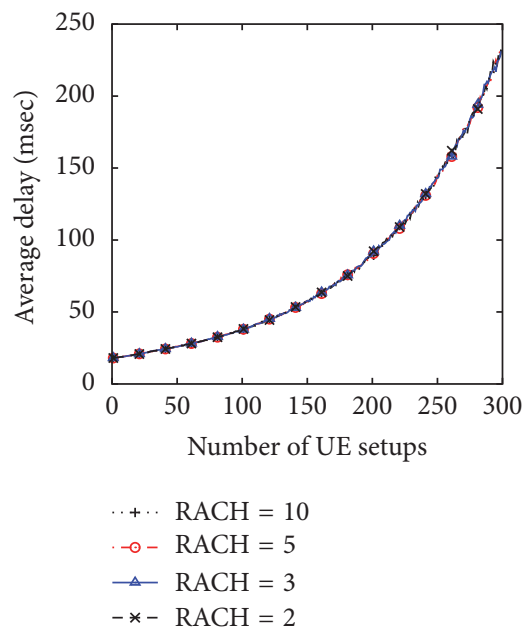

(b) CUL channel

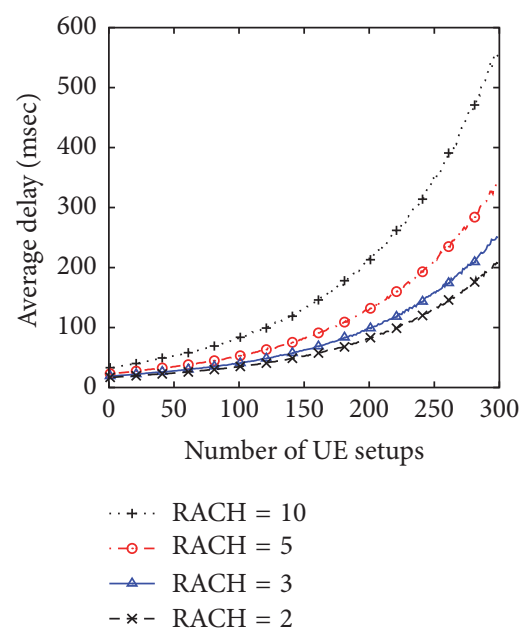

(c) CUL with RA

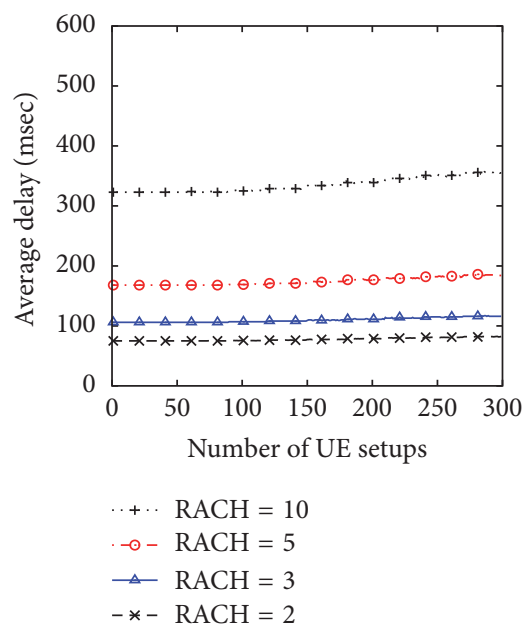

(d) Geo-location road based RA (GeoRA)

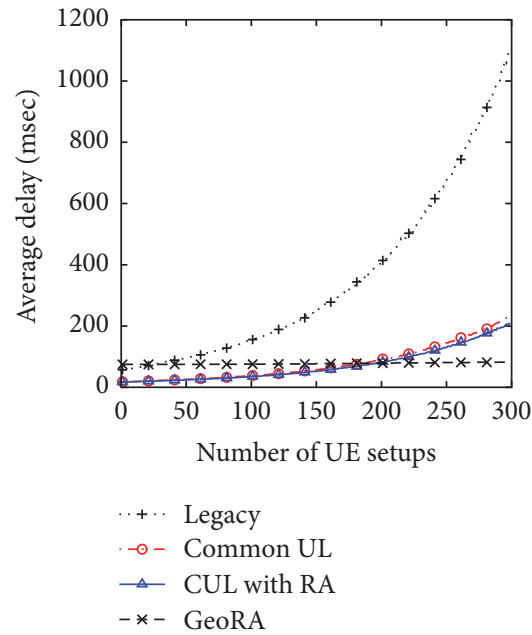

(e) Delay comparison with RACH $=2$

FIGURE 16: Average delay with varying period of RACH. 


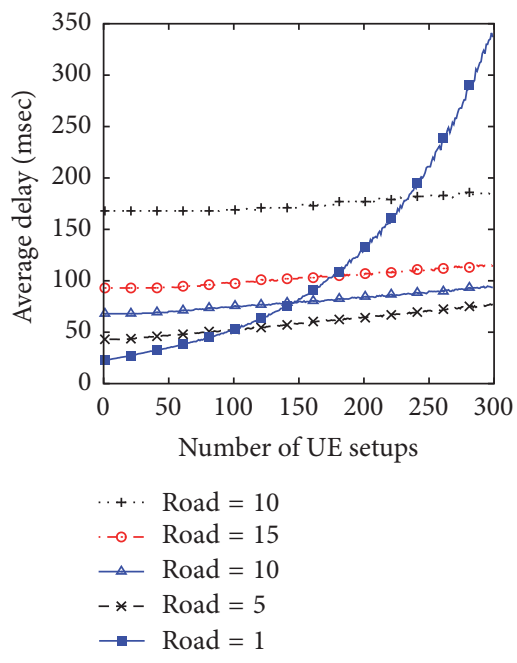

(a) Delay with number of roads

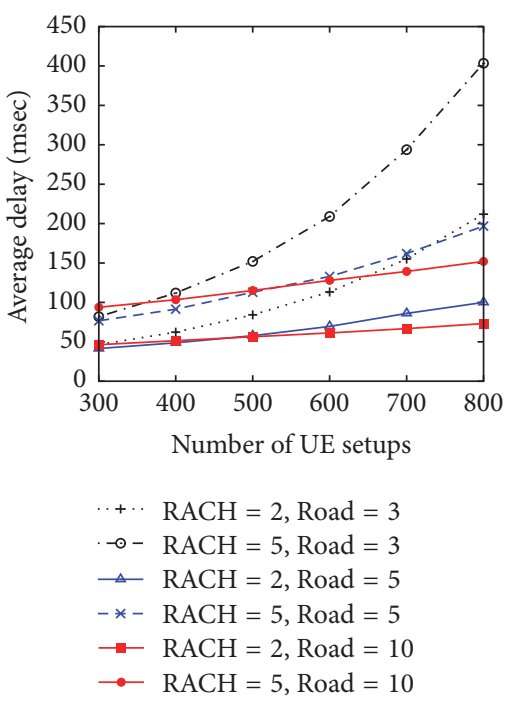

(b) More than 300 UE setups

FIGURE 17: Geolocation road based RA performance.

Consequently, the road segment group with size $=5$ achieves minimum delay, about $70 \mathrm{~ms}$ compared to other group sizes less than 300 UE setups. Large road segment groups that probably suffer collisions in the RACH have longer delay even though they have more frequent RACH opportunities. Since cell-based multicast and GCS AS delay are no more than $10 \mathrm{~ms}$, vehicular safety communication can be supported in LTE networks. However, more UE increase the delay in such large size group as can be seen in Figure 17(b). For example, the Road $=5$ shows longer delay than Road $=10$ with same $\mathrm{RACH}$ frequency $=5,150$ and $200 \mathrm{~ms}$ in 800 UE setups, respectively. To say, the $\mathrm{RACH}$ frequency is more critical than transmission collision among UE setups with large number of UE setups. Also, delay gap between different RACHs, 2 and 5 in same group size, supports that argument.

\section{Discussion}

Our approach still has many challenges to discuss in future works. Uplink transmission failure causes additional delay, which may require more RACHs. Otherwise, an additional mechanism can be considered to prioritize a random access for a retransmission by vehicles. For example, dedicated RACHs or preambles for retransmissions can be assigned.

Another issue is that the RACHs should be shared by normal human devices like smart phones and tablets and vehicles at the same time. It is burden to eNB that vehicles use $\mathrm{RACH}$ at every other subframe. Also, it leads to decrease RA performance of normal users. Thus, the eNB has to control a group size of road segments according to number of UE setups instead of increasing use of RACHs.

\section{Conclusion}

ITS is an attractive application for globally deployed LTE networks; however, the LTE system has not been fully investigated in terms of feasibility for safety purposes. Although several LTE system features are currently used for mission-critical group communications, they are inadequate for vehicular safety communication according to our analysis. We identified three major delay factors in LTE-based group communication and proposed corresponding solutions: a geolocation road based random access with a persistent uplink channel, a GCS AS on a mobile edge cloud, and a geolocation road based cell multicast.

From our simulation and analysis, we conclude that the total delay can be less than $80 \mathrm{~ms}$ with reasonable overhead of RACHs and persistent uplink channels; the uplink access takes $70 \mathrm{~ms}$, the cell multicast takes about $5 \mathrm{~ms}$, and the GCS AS may require an additional $5 \mathrm{~ms}$ processing delay. As a consequence, LTE networks using our enhanced features can support vehicular safety communications. Future works will include the investigation of efficient uplink channel scheduling for dynamically updated road topology and multiple cellbased broadcasts for fast-moving vehicles.

\section{Competing Interests}

The authors declare that there is no conflict of interests regarding the publication of this paper.

\section{Acknowledgments}

This research was supported by the National Research Foundation of Korea (NRF) funded by the Ministry of Science, ICT \& Future Planning (2016R1C1B1016084).

\section{References}

[1] D. Jiang and L. Delgrossi, "IEEE 802.11 p: towards an international standard for wireless access in vehicular environments," 
in Proceedings of the IEEE Vehicular Technology Conference (VTC Spring '08), pp. 2036-2040, 2008.

[2] S. U. Eichler, "Performance evaluation of the IEEE $802.11 \mathrm{p}$ wave communication standard," in Proceedings of the IEEE 66th Vehicular Technology Conference (VTC-Fall '07), pp. 2199-2203, 2007.

[3] ISO, "Intelligent transport systems $ł$ cooperative ITS $ł$ using V2I and $\mathrm{I} 2 \mathrm{~V}$ communications for applications related to signalized intersections," ISO/PDTS Std 19091, 2015.

[4] TR 22.885 v14.0.0, Study on LTE support for V2X services(Rel.14), 3GPP Std, 2015.

[5] ETSI, "Intelligent Transport Systems (ITS); Vehicular communications; Basic set of applications; Part 2: specification of cooperative awareness basic service," ETSI Std TS 102 637-3, 2010.

[6] ETSI, "TR 102962 intelligent transport systems (its); Framework for public mobile network," Tech. Rep., ETSI, 2012.

[7] G. Araniti, C. Campolo, M. Condoluci, A. Iera, and A. Molinaro, "LTE for vehicular networking: a survey," IEEE Communications Magazine, vol. 51, no. 5, pp. 148-157, 2013.

[8] M.-A. Phan, R. Rembarz, and S. Sories, "A capacity analysis for the transmission of event and cooperative awareness messages in LTE networks," in Proceedings of the 18th ITS World Congress, Orlando, Fla, USA, October 2011.

[9] K. Trichias, J. van den, G. Berg, J. de Jongh, and R. Litjens, "Modeling and evaluation of LTE in intelligent transportation systems," in Proceedings of the Joint ERCIM eMobility and MobiSense Workshop, D. Dimitrova, M. Brogle, T. Braun, G. Heijenk, and N. Meratnia, Eds., pp. 48-59, University of Bern, Bern, Switzerland, June 2012.

[10] A. Vinel, “3GPP LTE versus IEEE 802.11p/WAVE: which technology is able to support cooperative vehicular safety applications?" IEEE Wireless Communications Letters, vol. 1, no. 2, pp. 125-128, 2012.

[11] M. Kihl, K. Bur, P. Mahanta, and E. Coelingh, "3GPP LTE downlink scheduling strategies in vehicle-to-infrastructure communications for traffic safety applications," in Proceedings of the 17th IEEE Symposium on Computers and Communication (ISCC '12), pp. 000448-000453, Cappadocia, Turkey, July 2012.

[12] T. Mangel, T. Kosch, and H. Hartenstein, "A comparison of UMTS and LTE for vehicular safety communication at intersections," in Proceedings of the IEEE Vehicular Networking Conference (VNC '10), pp. 293-300, IEEE, Jersey City, NJ, USA, December 2010.

[13] Y. Yang, P. Wang, C. Wang, and F. Liu, "An eMBMS based congestion control scheme in cellular-VANET heterogeneous networks," in Proceedings of the 17th IEEE International Conference on Intelligent Transportation Systems (ITSC '14), pp. 1-5, IEEE, Qingdao, China, October 2014.

[14] W. Wu, P. Wang, C. Wang, and F. Liu, "A beacon rate control scheme based on embms in cellular-vanets heterogeneous networks," in Internet of Vehicles-Safe and Intelligent Mobility, pp. 324-335, Springer, Berlin, Germany, 2015.

[15] L.-C. Tung and M. Gerla, "LTE resource scheduling for vehicular safety applications," in Proceedings of the 10th Annual Conference on Wireless On-Demand Network Systems and Services (WONS '13), pp. 116-118, Banff, Canada, March 2013.

[16] J. Calabuig, J. F. Monserrat, D. Gozálvez, and O. Klemp, "Safety on the roads: LTE alternatives for sending ITS messages," IEEE Vehicular Technology Magazine, vol. 9, no. 4, pp. 61-70, 2014.
[17] ETSI, "Intelligent Transport Systems (ITS); Vehicular Communications; GeoNetworking; Part 3: network architecture," ETSI EN 302 636-3 V1.1.2, ETSI Std, 2014.

[18] K. Zheng, Q. Zheng, P. Chatzimisios, W. Xiang, and Y. Zhou, "Heterogeneous vehicular networking: a survey on architecture, challenges, and solutions," IEEE Communications Surveys \& Tutorials, vol. 17, no. 4, pp. 2377-2396, 2015.

[19] TS 37.868 RAN Improvements for Machine-type Communications, 3GPP Std.

[20] J.-P. Cheng, C.-H. Lee, and T.-M. Lin, "Prioritized random access with dynamic access barring for ran overload in 3gpp ltea networks," in Proceedings of the IEEE GLOBECOM Workshops (GC Wkshps '11), pp. 368-372, IEEE, Houston, Tex, USA, December 2011.

[21] T.-M. Lin, C.-H. Lee, J.-P. Cheng, and W.-T. Chen, "PRADA: prioritized random access with dynamic access barring for MTC in 3GPP LTE-A networks," IEEE Transactions on Vehicular Technology, vol. 63, no. 5, pp. 2467-2472, 2014.

[22] A. S. Lioumpas and A. Alexiou, "Uplink scheduling for machine-to-machine communications in LTE-based cellular systems," in Proceedings of the IEEE GLOBECOM Workshops (GC Wkshps '11), pp. 353-357, IEEE, Houston, Tex, USA, December 2011.

[23] M. Hasan, E. Hossain, and D. Niyato, "Random access for machine-to-machine communication in LTE-advanced networks: issues and approaches," IEEE Communications Magazine, vol. 51, no. 6, pp. 86-93, 2013.

[24] L. Gallo and J. Harri, "Short paper: A LTE-direct broadcast mechanism for periodic vehicular safety communications," in Proceedings of the IEEE Vehicular Networking Conference (VNC '13), pp. 166-169, Boston, Mass, USA, December 2013.

[25] A. Khelil and D. Soldani, "On the suitability of device-to-device communications for road traffic safety," in Proceedings of the IEEE World Forum on Internet of Things (WF-IoT '14), pp. 224229, IEEE, Seoul, South Korea, March 2014.

[26] A. Bazzi, B. M. Masini, and A. Zanella, "Performance analysis of $\mathrm{V} 2 \mathrm{v}$ beaconing using LTE in direct mode with full duplex radios," IEEE Wireless Communications Letters, vol. 4, no. 6, pp. 685-688, 2015.

[27] TS 23.246 Multimedia Broadcast/Multicast Service (MBMS); Architecture and functional description, 3GPP Std, http://www .3gpp.org.

[28] J. Huschke and M.-A. Phan, "An overview of the cellular broadcasting technology embms in lte," in Next Generation Mobile Broadcasting, p. 223, 2013.

[29] "TS 36.331 Evolved Universal Terrestrial Radio Access (EUTRA)," Radio Resource Control (RRC); Protocol specification, 3GPP Std.

[30] TS 22.468 Group Communication System Enablers for LTE (GCSE LTE), 3GPP Std, http://www.3gpp.org.

[31] TS 23.203 Policy and charging control architecture, 3GPP Std, http://www.3gpp.org/.

[32] TR 36.913 Requirements for further advancements for Evolved Universal Terrestrial Radio Access (E-UTRA), 3GPP Std, http://www.3gpp.org/.

[33] B. Lantz, B. Heller, and N. McKeown, "A network in a laptop: rapid prototyping for software-defined networks," in Proceedings of the 9th ACM SIGCOMM Workshop on Hot Topics in Networks, p. 19, Monterey, Calif, USA, October 2010.

[34] N. McKeown, T. Anderson, H. Balakrishnan et al., "OpenFlow: enabling innovation in campus networks," ACM SIGCOMM 
Computer Communication Review, vol. 38, no. 2, pp. 69-74, 2008.

[35] R. Bifulco, M. Brunner, R. Canonico, P. Hasselmeyer, and F. Mir, "Scalability of a mobile cloud management system," in Proceedings of the 1st ACM Mobile Cloud Computing Workshop (MCC '12), pp. 17-22, August 2012.

[36] L. E. Li, Z. M. Mao, and J. Rexford, "Toward software-defined cellular networks," in Proceedings of the 1st European Workshop on Software Defined Networks (EWSDN '12), pp. 7-12, IEEE, Darmstadt, Germany, October 2012.

[37] "Study on Support of single-cell point-to-multipoint transmission in LTE," TR 36.890 v13.0.0, 3GPP Std, 2015.

[38] 3GPP, “TS 36.300 Evolved Universal Terrestrial Radio Access (E-UTRA) and Evolved Universal Terrestrial Radio Access Network (E-UTRAN); Overall description; Stage 2," 3GPP Std, 2015.

[39] S. Kim, K. Jung, J. Choi, and Y. Kwak, "Improving LTE/LTE-A UE power efficiency with extended DRX cycle," in Proceedings of the 80th IEEE Vehicular Technology Conference (VTC '14), pp. 1-5, IEEE, Vancouver, Canada, September 2014.

[40] M. Behrisch, L. Bieker, J. Erdmann, and D. Krajzewicz, "Sumosimulation of urban mobility," in Proceedings of the 3rd International Conference on Advances in System Simulation (SIMUL '11), Barcelona, Spain, 2011.

[41] https://www.openstreetmap.org/.

[42] D. Eckhoff, N. Sofra, and R. German, "A performance study of cooperative awareness in ETSI ITS G5 and IEEE WAVE," in Proceedings of the 10th Annual Conference on Wireless OnDemand Network Systems and Services (WONS '13), pp. 196200, Banff, Canada, March 2013.

[43] Q. Xu, T. Mak, J. Ko, and R. Sengupta, "Medium access control protocol design for vehicle-vehicle safety messages," IEEE Transactions on Vehicular Technology, vol. 56, no. 2, pp. 499-518, 2007. 

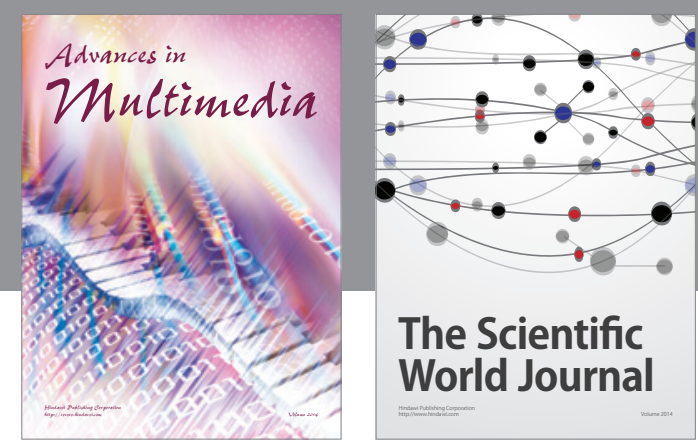

The Scientific World Journal
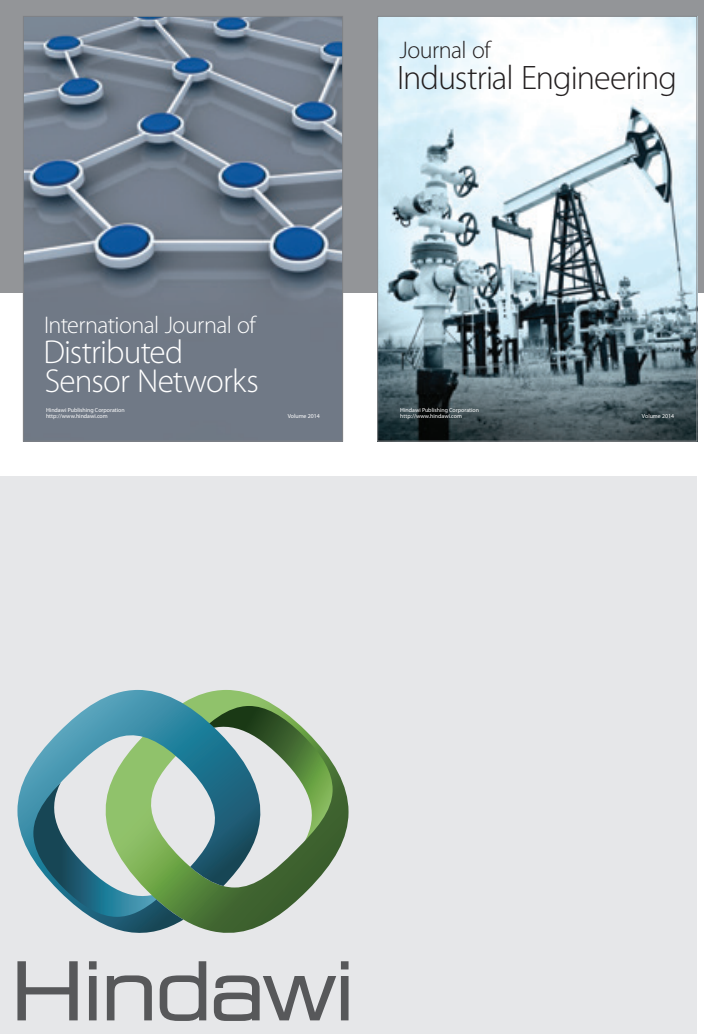

Submit your manuscripts at

https://www.hindawi.com

\section{Computer Networks} and Communications
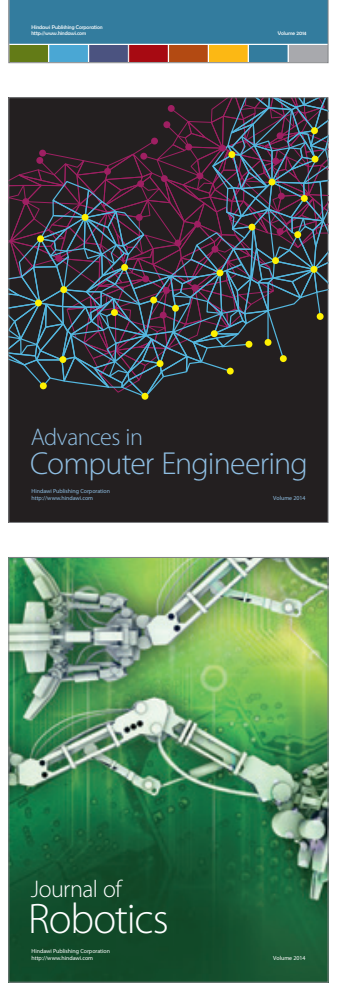
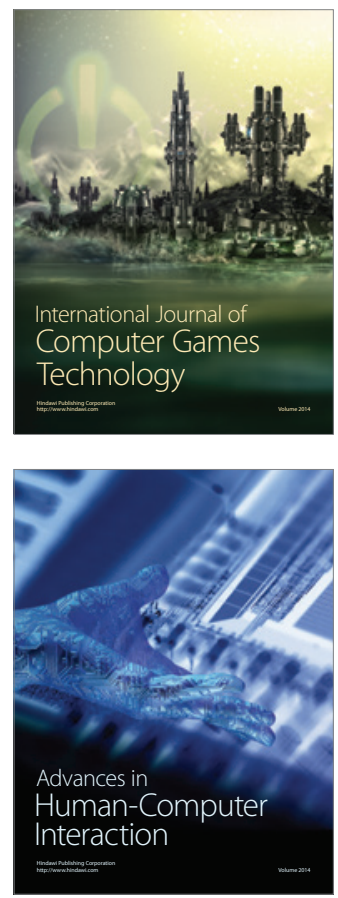
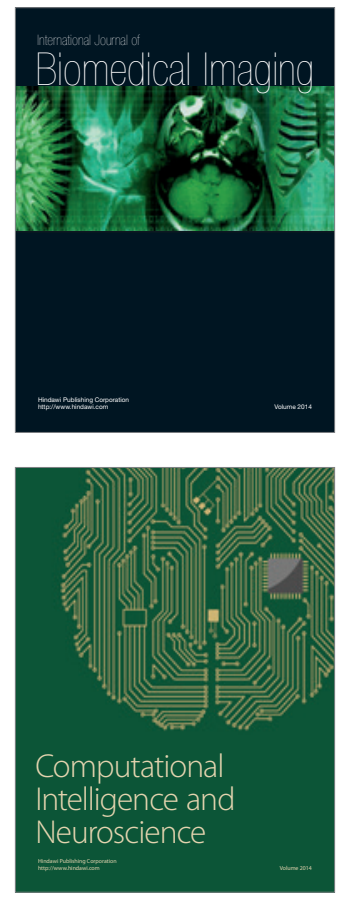
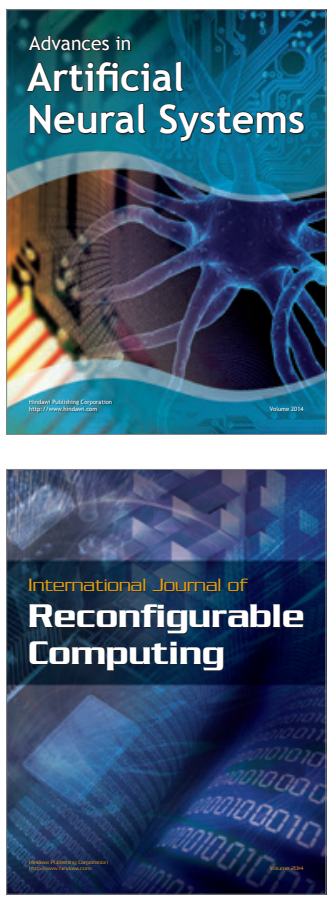
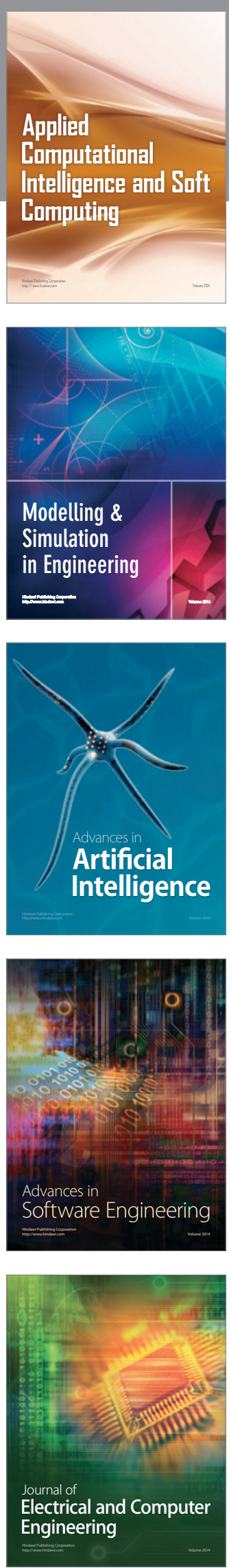